\title{
Sockets for limb prostheses: a review of existing technologies and open challenges
}

\author{
Linda Paternò, Michele Ibrahimi, Emanuele Gruppioni, Member, IEEE, Arianna Menciassi, Senior, \\ IEEE, and Leonardo Ricotti, Member, IEEE
}

\begin{abstract}
In the prosthetics field, one of the most important bottlenecks is still the human-machine interface, namely the socket. Indeed, a large number of amputees still rejects prostheses or points out a low satisfaction level, due to a suboptimal interaction between the socket and the residual limb tissues. The aim of this paper is to describe the main parameters (displacements, stress, volume fluctuations and temperature) affecting the stump-socket interface and reducing the comfort/stability of limb prostheses. In this review, a classification of the different socket types proposed in the literature is reported, together with an analysis of advantages and disadvantages of the different solutions, from multiple viewpoints. The paper then describes the technological solutions available to face an altered distribution of stresses on the residual limb tissues, volume fluctuations affecting the stump overtime and temperature variations affecting the residual tissues within the socket. The open challenges in this research field are highlighted and the possible future routes are discussed, towards the ambitious objective of achieving an advanced socket able to self-adapt in real-time to the complex interplay of factors affecting the stump, during both static and dynamic tasks.
\end{abstract}

Index Terms - amputee, biomechanics, liners, pistoning, pressure, prosthesis fitting, residual limb, shear stress, sockets, suspension systems, temperature, volume fluctuation.

\section{INTRODUCTION}

Limb amputations cause serious physical disabilities that compromise the quality of life of a large number of people, globally. The World Health Organization estimates that there are $\sim 40$ million amputees in the world [1]. This number is expected to further grow, due to an increased life expectancy and a corresponding higher incidence of diabetes and vascular diseases. Indeed, such pathologies are the most common amputation causes, followed by traumatic events and cancer [2].

Limb prostheses have the main purpose to reduce the negative impact of such disabilities, attempting to restore a

This work was supported by the project MOTU (robotic prosthesis with sMart sOcket and bidirectional inTerface for lower limb ampUtees), funded by INAIL (https://www.santannapisa.it/en/institute/biorobotics/motu). The authors would like to thank the staff of the "Centro Polifunzionale Piccioli" in Cascina (PI, Italy) for their contribution in the identification of the main issues involved in the socket design and use.

L. Paternò, M. Ibrahimi, *A. Menciassi and L. Ricotti are with the BioRobotics Institute, Scuola Superiore Sant'Anna, Pontedera (PI) 56025, Italy (correspondence e-mail: arianna.menciassi@ santannapisa.it).

E. Gruppioni is with the INAIL Centro Protesi, Vigorso di Budrio (BO) 40054, Italy. normal functionality and amputee autonomy, as much as possible. In order to achieve functional and comfortable prostheses, great attention has to be paid to the socket design. In fact, the socket represents a critical interface between the user (natural) stump and the prosthetic (artificial) device. A suitable socket has to ensure efficient fitting, appropriate load transmission, stability and control [3] and it often constitutes a key factor for the success or failure of the prosthesis itself.

At present, despite some important recent advances in prosthetics, several amputees still reject their prostheses or show a rather low satisfaction level. This is mainly due to socket-related issues, such as poor comfort, reduced biomechanical functionality and hampered control [4]. In addition, skin lesions occur in the $63-82 \%$ of lower limb amputees, thus causing a prosthesis abandon rate that is around $25-57 \%$ [5].

In this framework, the factors playing a key role can be depicted as in Fig. 1. Obviously, the socket shape and its constitutive materials are of paramount importance, as well as the suspension system, which affects the stability of the residual limb within the socket. The suspension can be applied by leaving the stump in direct contact with the socket or by applying a liner (a flexible soft cover) (Fig. 2).

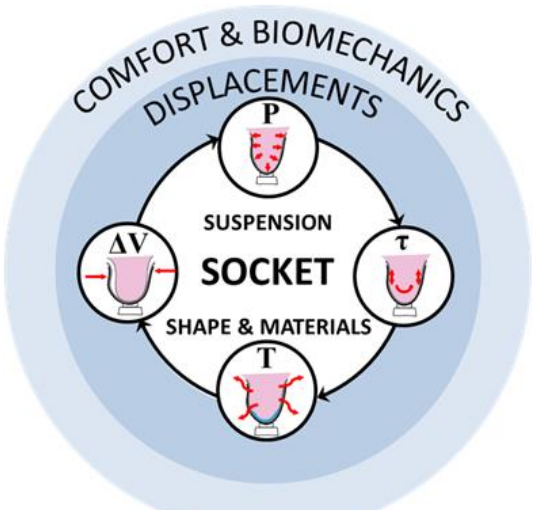

Fig. 1. Schematic representation of the main factors affecting the stumpsocket interface and their interplay (suspension $=$ system used to guarantee the adhesion of the residual limb to the socket; $\mathrm{P}=$ pressure; $\tau=$ shear stress; $\mathrm{T}=$ temperature; $\Delta \mathrm{V}=$ volume fluctuations; displacements = relative movements between the stump and the socket).

A specific combination of socket shape and materials, together with a specific suspension system, determines the distribution of pressures and shear stresses on the tissues, as well as the temperature and volume fluctuations affecting the residual limb. All these parameters are interconnected: volume 
fluctuations may imply a reduced prosthesis fitting, but also an altered distribution of pressures and shear stresses on the limb residual tissues. This can lead to rubbing phenomena that, in concert with the barrier constituted by socket and liner, alter the thermoregulation system by producing sweating, irritation and smell.

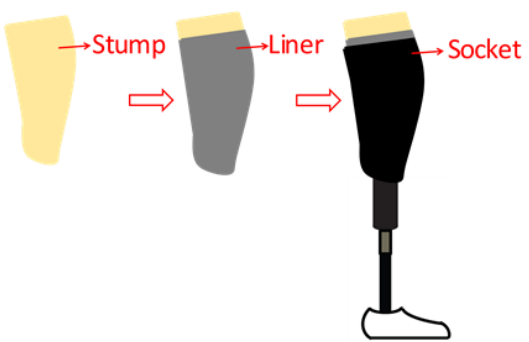

Fig. 2. Representation of a socket provided with a liner between it and the stump. The liner role is normally to increase the stability between the stump and the socket.

All these parameters typically play a role in enhancing the relative displacement between the stump and the socket. In lower limb amputees, the so-called "pistoning effect" occurs. It consists of a vertical relative movement of the stump respect to the socket, sometimes associated with abrasive phenomena. Overall, all the mentioned parameters and effects may hamper the prosthesis comfort and biomechanical stability. Optimizing the interaction between the stump and the inner surface of the socket is thus essential to guarantee the long-term acceptability, the comfort and a good functionality of the prosthesis.

Despite the recent research efforts in this field, no fully satisfactory technological solutions exist at present. A comparison between the existing sockets, analyzing their merits and drawbacks, has been made by Safari et al. [6], AlFakin et al. [7] and Chatterjee et al. [8] concerning transtibial prostheses.

The purpose of this paper, which makes it different from the existing reviews on this topic, is to make a complete overview of the existing sockets for lower limb prostheses, with some links to upper limb prostheses, when relevant (see the online supplement - Annex 1). Indeed, for upper and lower limb amputees the socket-related issues are often the same, although with a different impact. As a consequence, the possible technological solutions are similar, in many cases.

After a brief description of different existing socket designs, the next sections analyze the key parameters that influence the efficiency and acceptability of a socket. Displacements, interfacial stresses, volume fluctuations, and thermal discomforts are presented, describing also the related health issues, as anticipated in the review by Mak et al. [3], but also reporting quantitative outcomes, and discussing the possible engineering approaches that may generate effective solutions. The open challenges are then identified and discussed.

\section{SOCKET SHAPE AND BIOMECHANICS}

To date, socket design and suspension system are chosen by the prosthetist, based on the amputees' residual limb features (mainly stump dimensions and muscular trophism), on the patient age, lifestyle and activity level. This process is still often based on the prosthetist's personal experience, rather than on objective data. Fig. 3a shows the terminology and the abbreviations typically used to describe a socket and its interaction with the stump tissues.

(a)

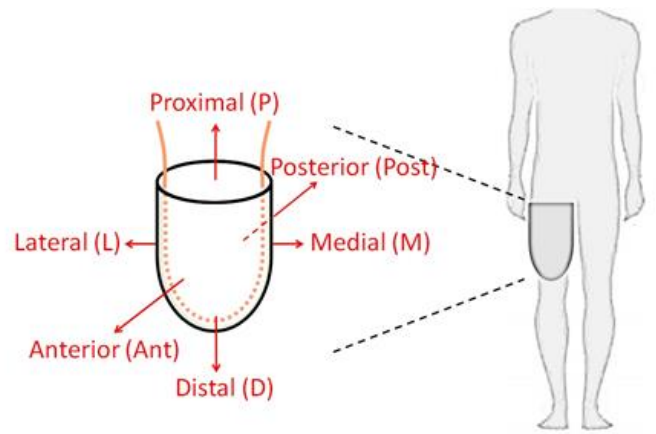

(b)

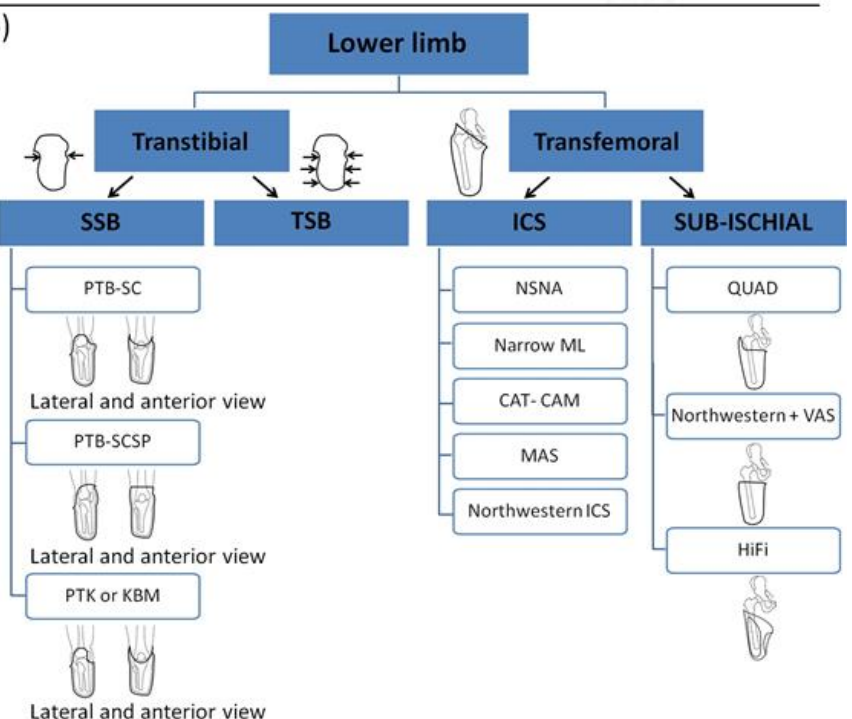

Fig. 3. a) Schematic representation of the different anatomical positions typically used to describe the interaction between socket and tissues. b) Prosthetic socket classification for lower limb $(\mathrm{SSB}=$ Specific Surface Bearing; TSB = Total Surface Bearing; ICS = Ischial Containment Sockets; PTB = Patellar Tendon Bearing; $\mathrm{SC}=$ SupraCondylar; SCSP = SupraCondylar SupraPatellar; PTK = Patellar Tendon Kegel; $\mathrm{KBM}=$ Kondylen-Bein-Muenster; NSNA $=$ Normal Shape-Normal Alignment; narrow $\mathrm{ML}=$ narrow Medio-Lateral; $\mathrm{CAT}-\mathrm{CAM}=$ Contoured Adducted Trochanteric-Controlled Alignment Method; MAS = Marlo Anatomical Socket; QUAD = quadrilateral; VAS = Vacuum Assisted Socket).

Concerning lower limbs (Fig. 3b), the first studies on socket design were carried out in the early '50s [9]. The so-called Specific Surface Bearing (SSB) sockets and in particular the Patellar Tendon Bearing (PTB) ones, became the first standards for transtibial amputees. They were featured by a medio-lateral grip on femoral condyles and were developed in two versions: the supracondylar (PTB-SC) one and the supracondylar/suprapatellar (PTB-SCSP) one. The latter had a more extended anterior wall that fully covered the patella [9]. Another design type owning to the SSB family was the Patellar Tendon Kegel (PTK), also called Kondylen-BeinMuenster (KBM) socket, featured by higher proximal mediallateral walls compared with the previously mentioned systems. The main aim of SSB sockets is to apply loads on specific regions, which are typically more tolerant to high pressure values. However, by reducing the overall anatomical area 
loaded, such regions have inevitably to support high pressure values, which can cause ulcers and other skin problems. This was the main motivation for the introduction of Total Surface Bearing (TSB) sockets in the ' 80 s. These systems aimed at distributing the load on the total stump area [10], avoiding high local stresses and enhancing the comfort and fitting, as well as the overall proprioception.

Concerning transfemoral amputees, the sub-ischial quadrilateral socket (QUAD), a SSB socket featured by ischial weight bearing and a quadrilateral shape in the transverse plane, was the most adopted solution until the late '90s. More recently, the QUAD design was mostly replaced by Ischial Containment Sockets (ICS). They can be developed in different configurations: the Normal Shape-Normal Alignment (NSNA) one [11], the narrow Medio-Lateral (ML) one [12], the Contoured Adducted Trochanteric-Controlled Alignment Method (CAT-CAM) one, the Marlo Anatomical Socket (MAS) one and the Northwestern ICS [13]. Most of them are TSB sockets and improve the alignment of the femur and the prosthesis axes, thus enhancing the medial-lateral stability. This is normally achieved through extended medial-lateral brimlines, which contain the ischial tuberosity and ramus.

Recently, the sub-ischial design has been proposed again with two recent solutions: the sub-ischial Northwestern [14] and the High Fidelity (Hi-Fi) sockets [15]. They constitute new solutions for medium-long stumps, aiming to enhance stability, gait and comfort. The sub-ischial Northwestern socket exploits Vacuum Assisted Suspensions (VAS, described more in detail in section III) to guarantee stability. The Hi-Fi socket is based on a frame of 3-4 struts that extend longitudinally and compress the limb (Fig. 4) [15]. The fenestrations allow tissues to slightly stick out. The compression areas stabilize the bone, reduce the motion with respect to the bone and lock the skin.

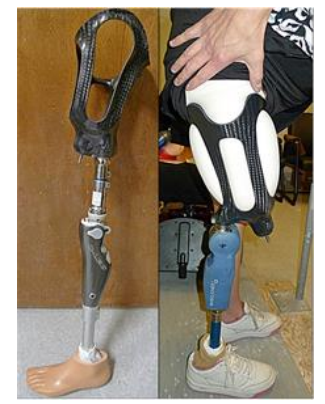

Fig. 4. HiFi sockets for the lower limbs (reproduced with permission of the U.S. Department of Veterans Affair [15]).

\section{A. Biomechanical parameters in lower limb amputees}

Quantitative biomechanical analyses allow to evaluate the effectiveness of different socket designs and how they affect the overall prosthesis biomechanics. The investigated parameters include energy expenditure, range of motion (ROM), velocity, cadence, stride length, stride width, percentage of swing, stance, etc. Energy expenditure can be mainly measured by the $\mathrm{VO}_{2}$ (i.e. the maximum oxygen volume consumed per minute, per $\mathrm{kg}$ of body weight at maximum performance) and the $\mathrm{VCO}_{2}$ (an analogous parameter for $\mathrm{CO}_{2}$ emission). Motion capture systems are the most used tools to get kinematic information. However, they imply the need of a structured and standardized environment. As a consequence, the evaluation of daily activities are highly sub-investigated.

In general, $52.4 \%$ of lower limb amputees report falling accidents ( $40.4 \%$ with injuries), and $49.2 \%$ of them report fear of falling [16]. Falls and comorbidities can alter the energy consumption of walking and the overall gait speed [17], [18].

In the review of Safari and Meyer [6] on transtibial amputees it is shown that TSB sockets improve gait symmetry and further symmetry can be achieved with VAS.

Concerning transfemoral sockets, Radcliffe et al. [19] clarified that the main biomechanical issue affecting the QUAD systems is the proximal shifting in the medial-lateral direction. This implies the femur hitting at the distal lateral wall, caused by pelvis rotation towards the medial side. The abduction angle of a QUAD socket was also confirmed by Long et al. [11]. A comparison between QUAD and ICS sockets in terms of energy consumption was made by Flandry et al. [20], reporting a lower energy expenditure $(-56 \%)$ in the latter configuration. In addition, Gailey et al. [21] showed a $20 \%$ lower energy expenditure when using the CAT-CAM socket, with respect to the QUAD one.

Recent efforts focused on the effects of brim removal on the $\mathrm{Hi}-\mathrm{Fi}$ and the sub-ischial Northwestern systems. Results showed gait parameters at least comparable or even more symmetrical than standard ICS systems. Thus, brimless sockets were claimed as preferable [6], [22]. Kahle et al. [23] compared ICS to Hi-Fi sockets and found a greater mean distance walked for the latter systems. The amputees also compiled a report, in which they confirmed an increased balance confidence during daily activities guaranteed by the Hi-Fi socket [22], [23]. Alley et al. reported an improvement in cadence and step length for a transfemoral amputee using the Hi-Fi sockets, in comparison with a traditional ICS system [24].

A specific socket design can help to overcome biomechanical issues caused by the amputation. However, they are also affected by the suspension system and prosthesis components, as well as by the alignment of the prosthesis itself [25]. Inadequate prosthesis components can increase the metabolic cost, can wrongly activate muscles, and finally can decrease the gait symmetry. For this reason, many studies attempted to understand the mechanical behavior of prosthetic ankle-foot devices and their biomechanical implications [26]. Prosthetic feet must guarantee stability, especially during the early stance phase, allowing an adequate knee flexion moment, and during the late stance phase, allowing propulsion during the forward locomotion of the contralateral limb [27] [26]. This can be achieved through variable stiffness components with an efficient energy return, thus providing high torque, and paying attention to alignment issues. It has been found that dynamic feet (e.g. systems able to store and release energy during the walk) lower the user energy expenditure and improve gait, even if not significantly [28]. More recent efforts have been directed to add active components in prosthetic feet to mimic the ankle-foot natural 
movement [29]. However, their use is still limited since they are heavy and bulky, and require high mental efforts during use [30]. In this framework, the most relevant challenge is the set-up of control algorithms able to guarantee a high reliability keeping the mental effort low [31].

Traditional pylons (i.e. the connection elements between prosthetic foot and knee, or foot and socket) were rigid elements. Nowadays, Vertical Shock Absorbing Pylons (VSAPs), can absorb cyclic loads applied on tissues, thus decreasing the shock transmitted to the socket [32]. Their mechanical behavior has to be studied more in deep, but a certain reduction of user energy expenditure has been demonstrated [33].

A transverse rotation adapter, adjustable by the prosthetist, can be placed at the base of the socket or between the pylon and the foot to rotate all the assembly [33]. This facilitates simple tasks, such as wearing a shoe, and lowers the shear stresses on the skin, by allowing micro-rotation of the components in the transversal plane [34]. It has been found that these devices can reduce the metabolic cost and improve the activity level of the user [34]. Recently, a variable stiffness rotator has been developed by Pew and Klute [34], demonstrating a reduction of shear stresses applied on stump tissues by regulating the stiffness in the transverse plane.

The knee is a key component to guarantee a good prosthesis stability. In particular, it is important in the early stance phase to absorb the vertical shocks, in the midstance to guarantee a lowered center of mass and in the late stance in order to guide the initial swing phase of the artificial leg, thus avoiding the tip-toe hitting on the ground [27]. Nowadays the more efficient solutions are the MicroProcessors Knees (MPKs), which allow more natural gait, improving the prosthesis efficiency and stability, and reducing the oxygen consumption [25]. In these advanced systems, the on-board microprocessor control unit detects the different gait phases of the user by means of load and joint angle sensors, accelerometers and gyroscopes and consequently adjusts the system resistance, to increase the user confidence [27].

When all prosthetic components are chosen, another crucial aspect is their alignment, which determines gait stability and fluency. This aspect has to be optimized considering the rotation and translation of each component in the different planes, according to the physiological lines. The state-of-theart about alignment variations in transtibial amputees has been reviewed by Neumann et al. [35] and Davenport et al. [36]. They evidenced that a wrong alignment can affect several kinematic parameters, as well as the pressure distribution at the stump/socket interface. Schmalz et al. [25] verified that an increased energy expenditure is required in case of a wrong alignment and this effect results more significant for transfemoral amputees, than for transtibial ones. Furthermore, it has been found that a wrong alignment in transfemoral amputees, causes an increased hip extension moment during the early stance phase, trunk flexion, a decreased step length and other undesired biomechanical effects [37].

\section{SUSPENSION SYSTEMS AND PROSTHESIS FITTING}

\section{A. Suspension systems for lower limbs}

The prosthesis fitting is mainly determined by its design and its suspension system. Thus, suspensions are one of the key factors that influence user satisfaction, as they guarantee the adhesion of the residual limb to the socket.

Several suspensions are available at present (Fig. 5).

The simplest one, which has been used since the $20^{\text {th }}$ century, is a harness, made of rigid or elastic belts. Belt suspensions are not a stable solution per se, and can act in synergy with other suspension systems.

Another possible solution is the subatmospheric suspension, based on the regulation of negative pressures values within the socket. This is the most diffused for lower limb prostheses. Negative pressures can be created by skin suction (e.g. skin-fit suction), through a unidirectional valve that allows the expulsion of the residual air. This system allows the maximum user proprioception, but increases the risk of skin problems. Roll-on liners are based on alternative mechanisms that maximize stability: they can be fixed to the socket through a pin-lock, a magnetic-lock or a lanyard strap (Fig. 6).

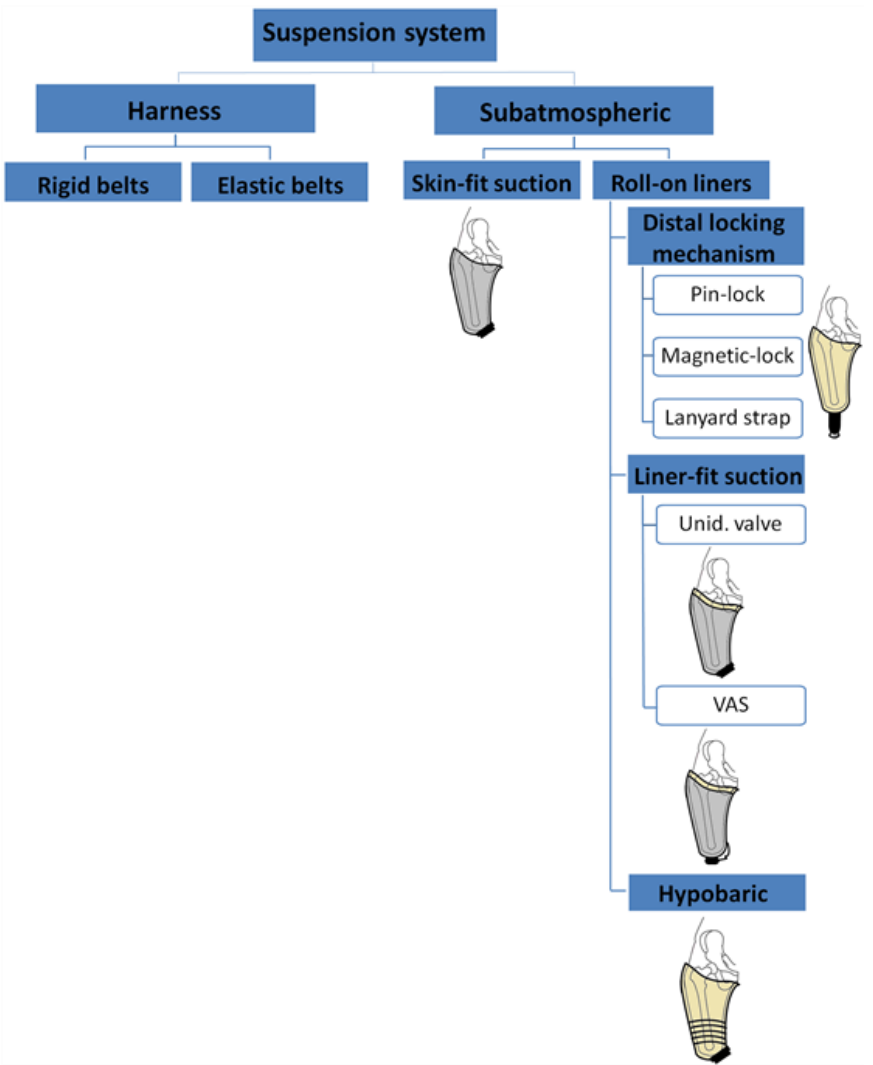

Fig. 5. Classification of socket suspension systems. The figure depicts such systems for transfemoral amputees, but they can be applied to all socket types.

Liners are extensively used in the standard practice, due to their ability to adhere to the skin, to form a protection against abrasion and to distribute loads [38]. In the past, prosthetic liners were made of open and closed cell foams, such as Pelite. Nowadays, silicone or other elastomers-based liners, rolled on the residual limb, allow higher durability and a more effective cushioning effect [9]. Bench tests suggest that elastomeric gel liners allow reduced shear stress on the skin and higher cushioning effect on bony prominences, since they are rather soft, in compression, and similar to biological tissues. [38] [9]. However, they usually increase perspiration, reducing the 
hygiene and causing dermatitis or other skin irritations. Urethane liners guarantee a better skin adhesion, thus avoiding skin breakdowns, thanks to their high friction coefficient, while silicone elastomers provide a better suspension thanks to their higher stiffness in tension and are thus preferable for softer stumps, in order to minimize the relative displacement between the socket and the residual limb tissues.

Roll-on liners can be also associated with an air evacuation system based on a unidirectional valve or with a VAS system (liner-fit suction), allowing a better fitting in comparison with distal locking mechanisms. Finally, hypobaric seals can be used, coupled with valves or VAS (Seal-In liner in Fig. 6a).

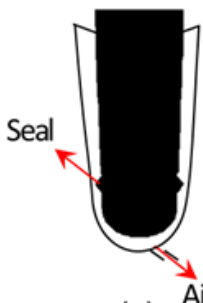

(a)

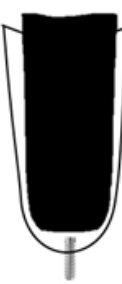

(b)

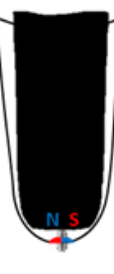

(c)

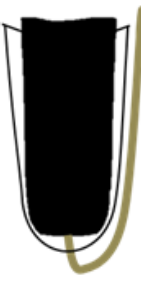

(d)
Fig. 6. From left to right: Seal-In (a), Pin-lock (b), Magnetic-lock (c), Lanyard strap (d) (reproduced with permission from MDPI [7]).

VAS systems apply a subatmospheric environment through a mechanical or an electrical activation means [27], [14], [39]. They reduce the motion between the residual limb and the socket, thus also reducing pistoning events, increasing the prosthesis control [22], [40]-[42] and allowing lower socket brims [14]. They are also considered to improve wound healing and reduce volume fluctuations [42], [43]. Hence vacuum suspensions could be a valid solution. On the other hand, VAS increase socket weight, need maintenance and can raise difficulties in donning, as highlighted in some cases [40].

\section{B. Displacements in lower limb amputees}

The efficacy of a suspension system is normally evaluated by measuring the relative displacements between the stump and the socket. In lower limbs the main displacement-related event is the "pistoning effect". Pistoning, meant as the vertical movement of the stump inside the socket, can be relative to the skin-socket, bone-socket or liner-socket interface. It can be measured through different systems, such as radiography, ultrasound, computerized tomography, standardized photographies and motion analysis systems based on markers [39], [44]. It can be measured in static conditions or in dynamic ones, by applying or not applying a load on the amputee's prosthetic leg (Table I).

For transtibial amputees, Baars et al. [45] demonstrated that liner-TSB sockets were less affected by pistoning respect to PTB sockets. Yiğiter et al. [46] quantified the pistoning effect for PTB and TSB sockets, which was $16 \pm 4 \mathrm{~mm}$ and $4 \pm 5$ $\mathrm{mm}$, respectively. Recently, pistoning differences between Dermo pin-lock liners and Seal-In liners has been investigated in TSB sockets, reporting a higher user satisfaction with the Seal-In liners [12]. Brunelli et al. confirmed that Seal-In liners guaranteed a reduced pistoning in comparison with traditional liners [47].

Magnetic-lock suspensions have been recently introduced in the clinics. They seem to be easier to don/doff and show reduced noise. However, they are affected by a larger pistoning effect, in comparison with pin lock and suction systems [48]. Only a few works reported an objective observations of VAS, in terms of pistoning. Klute et al. [49] reported values for the limb-socket displacement, showing that TSB sockets with a pin-lock liner showed a significantly larger displacement in comparison with sockets provided with VAS. However, this work only analyzed static pistoning. As reviewed by Eshragi et al. [44], only 5 out of 18 articles have reported pistoning in dynamic conditions, for transtibial amputees.

It can be inferred that both Seal-In liners and vacuum suspensions imply a reduced pistoning, thus avoiding 'milking' [12] (e.g. an excessive elongation of distal tissues). However, Seal-In liners still show drawbacks, mainly related to their difficult donning/doffing [50], [51]. A survey on 90 transfemoral amputees reported a higher satisfaction for SealIn liners than common suction suspensions during fitting, sitting, and donning/doffing. This was mainly due to a reduced sweating, less pain and irritation, less pistoning effect, reduced swelling and reduced smell [39].

Only 2 papers evaluated static pistoning in transfemoral amputees: Convery and Murray [52] reported displacements on QUAD sockets, while Kahle et al. [41] analyzed subischial and ICS sockets. No papers are available on dynamic measurements regarding transfemoral amputees.

In conclusion, more experimental evaluations are needed in this domain, especially concerning transfemoral amputees, and a special attention should be paid to dynamic conditions.

The friction coefficient between skin and socket/liner (or between liner and socket) is extremely important to reduce pistoning, but a reliable and univocal clinical value still has to be found [3]. If the friction coefficient is too low, slippage between skin and socket/liner can occur. However, if the friction coefficient is too high, high shear stresses can be generated, with possible tissue distortion during donning/doffing and ambulation, and higher risks of skin breakdown. This motivates the scientific efforts dedicated to analyze frictional properties of human skin in relation with materials used into sockets, as reviewed by Mak et al. [3].

TABLE I

PISTONING VALUES IN LOWER LIMB AMPUTEES

\begin{tabular}{|c|c|}
\hline \multicolumn{2}{|c|}{ Transtibial } \\
\hline TSB + sleeve & $7.5 \pm 4.7 \mathrm{~mm}$ (unweighted bearing) \\
suspension [47] & $12.4 \pm 5.6 \mathrm{~mm}$ (30 N distal loading ) \\
\hline TSB + pin-lock & $5.4 \pm 0.6 \mathrm{~mm}$ (max value during gait) \\
liner [50] [48] & $5.8 \pm 0.8 \mathrm{~mm}$ (90 N distal loading) \\
\hline TSB + Seal-In & $2.5 \pm 0.4 \mathrm{~mm}$ (max value during gait) \\
liner [50] [48] & $2.8 \pm 0.5 \mathrm{~mm}$ (90 N distal loading) \\
\hline TSB + VAS [83] & $1 \mathrm{~mm}$ (liner) and 33 $\mathrm{mm}$ (tibia) (during axial \\
loading) \\
[49] & $1 \pm 3 \mathrm{~mm}$ (weighted / unweighted bearing) \\
\hline PTB [46] & $16 \mathrm{~mm}$ (stance / swing phase) \\
\hline Other types & N. A. \\
\hline \multicolumn{2}{|c|}{ Transfemoral } \\
\hline QUAD [52] & $40 \pm 0.5 \mathrm{~mm}$ \\
\hline Brimless [41] & $14 \pm 8 \mathrm{~mm}$ \\
\hline ICS [41] & $25 \pm 9 \mathrm{~mm}$ \\
\hline Other types & N. A. \\
\hline \multicolumn{2}{|c|}{} \\
\hline
\end{tabular}




\section{INTERFACIAL STRESSES}

\section{A. Pressure and shear stresses: general considerations}

Stress distribution at the interface between the limb and the prosthesis can generate many discomforts. High pressures applied on the skin for long periods and in a non-uniform way, can cause pressure ulcers, sensitive skin, irritations and partial or total vascular occlusions [5]. These phenomena alter the blood perfusion and the metabolic demand, thus causing an increase in temperature, sweat and tissue epidermis maceration [3] [53]. Furthermore, the friction between limb and socket produces shear stresses, which lead to tissue deformation and increase the risk of injuries [3], [10]. Skin problems can evolve in chronic infections requiring, in the worst cases, a re-amputation [54]. Identifying pressure threshold values can be the first step to reduce such discomfort [55]. This is crucial for lower limb sockets, in which the stumps are cyclically subjected to high stresses during gait.

Although the relationship between stresses and discomfort varies for each subject depending on weight, residual muscle tonicity and life style [56], there are stump areas that are more tolerant to high pressure values, such as the midpatellar tendon and the medial tibial ones in transtibial amputees, while other areas are more critical, such as the distal end of the stump, which is indeed left unloaded in the socket design [55].

In the state of the art, pain-raising pressure thresholds for the popliteal fossa and for the patellar tendon have been quantified as $\sim 50 \mathrm{kPa}$ and $\sim 120 \mathrm{kPa}$, respectively [57]. A value of $40 \mathrm{kPa}$ has been considered acceptable during sitting in healthy subjects [41]. However standard threshold values for the stress interface distribution within a socket are not available yet, also because they depend dramatically on the status of the patient residual tissues and on the prosthesis features. A step forward could be achieved through the use of multi-indenting devices, able to characterize the hyperviscoelastic properties of residual limb tissues and to investigate pain thresholds and tolerances for different anatomical areas [58].

Several studies have been dedicated to quantify the stress distribution in different prosthetic sockets for transtibial amputees, typically subjected to relatively high pressures. On the other hand, really few data are available on transfemoral patients. These efforts aimed at comparing different designs and suspension systems (see the online supplement - Annex 2). In general, all these studies are based on a rather small number of subjects and do not often rely on standardized procedures. It must be mentioned that interfacial stresses can change overtime, together with changes in the residual limb shape and tissue properties. This obviously depends also on the socket general features and its material properties.

Finite Element Analysis (FEA) simulations are a useful tool that partly circumvents the issue of performing extensive measurements. An efficient model could be even able to predict and optimize the effects of different socket designs, suspensions and materials, as reviewed by Dickinson et al. [59].

\section{B. Sensing technologies for measuring stress distribution in amputees}

As reviewed by Al-Fakih et al. [7], pressure sensors dedicated to stress distribution measurements within sockets can be piezo-resistive, strain gauges, capacitive or optical ones. They can be positioned by using dedicated holes or pistons through the socket wall, in direct contact with the skin (Fig. 7a) or the liner (Fig. 7b). Alternatively, they can be inserted (Fig. 7c) or embedded (Fig. 7d) into the socket.

To date, the piezo-resistive F-Socket System (Tekscan Inc., Boston, MA, USA) (Fig. 7e) is the most used commercial solution for performing pressure measurements in clinical settings. Piezo-resistive sensors can be designed in thin and flexible configurations, able to map the stress distribution on large areas of the stump. Nevertheless, these sensors can quantify only pressure values. In order to evaluate both normal and shear stresses, strain gauges can be adopted. They allow measurements with high sensitivity, but such measurement can be achieved only in few isolated points. The stiffness of these systems causes crosstalk and boundary problems due to stress concentrations, especially in the curved areas. Capacitive sensors may overcome these limitations. They can measure both shear and normal stresses through flexible systems, featured by better performances than piezo-resistive ones, especially in terms of drift and hysteresis [60] [61]. The only commercial capacitive sensor, currently used in the prosthetics field, is the Novel System (Novel Electronics Inc.,

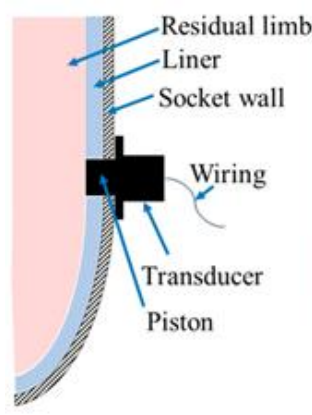

(a)

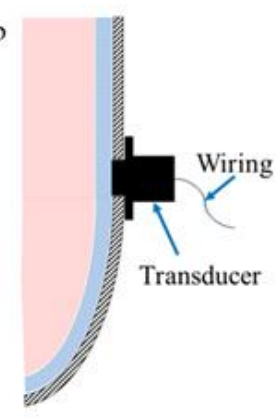

(b)

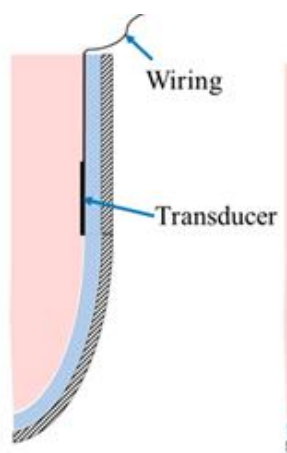

(c)

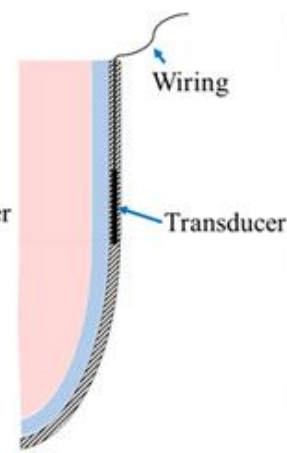

(d)

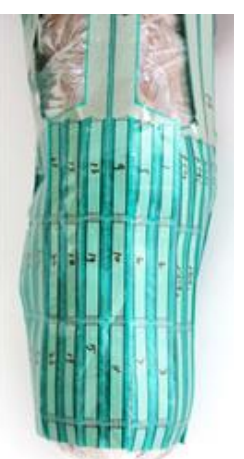

(e)

Fig. 7. Possible positioning strategies for sensors dedicated to stress measurements within sockets (piston sensor in contact with the skin (a), or with the liner (b), flexible sensor inserted within the liner in contact with the skin (c), or embedded within the socket wall in contact with the liner (d)); the F-socket sensor (e) (reproduced with permission from MDPI [7]). 
Saint Paul, MN, USA). However, it only measures pressure. The main problems of capacitive transducers are their need for a rather complex electronics, a non-negligible crosstalk noise within matrix array configurations and a high cost.

Opto-electronic systems (Fig. 8a) or fiber bragg gratings (FBGs, Fig. 8b) may allow the evaluation of normal and shear stresses with high sensitivity. They have been recently embedded in a liner or in a socket [62]-[65]. The main disadvantage of these systems is the relatively high risk of damaging the fibers or the electronic components. Therefore, their application in prosthetic sockets is limited yet and more studies are required to confirm the opportunity to exploit them in this field.
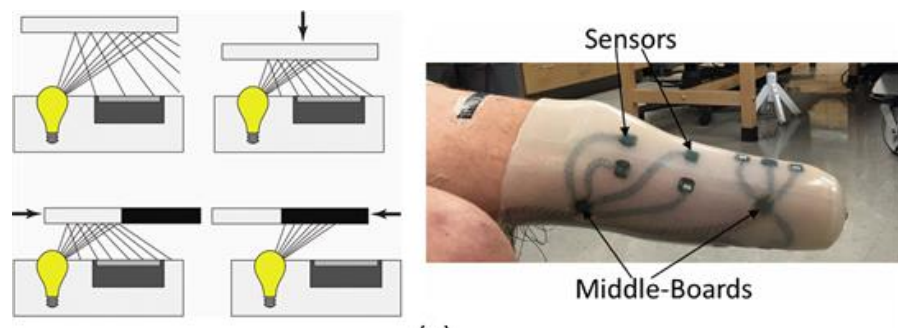

(a)

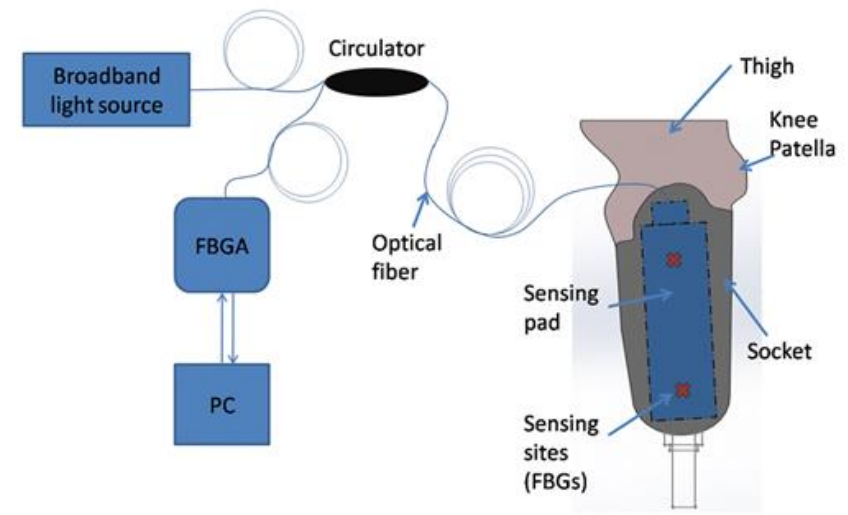

(b)

Fig. 8. Opto-electronic sensors integrated into the liner (a) and sesorized pads with fiber bragg gratings integrated into the socket (b). Images reproduced with permission from IEEE [62], [63]

An alternative solution to overcome the issues related to sensing technologies for prosthetic sockets is an approach based on artificial intelligence. To this purpose, an artificial neural network (ANN) was applied, combining experimental and numerical data [67]-[69]. In this way, it was possible to predict the pressure distribution inside a socket, starting from the data obtained through strain gauges located on the outer surface. During the training phase, a large amount of data were collected, by applying pressures on the internal socket surface (ANN input data) and collecting the related strain at the outer surface (ANN output data). Then, through a nonlinear transfer function, surface strain data allowed to predict the interfacial pressure values without socket modifications and without interfering with the interfacial conditions, differently from the sensing technologies described above. This promising solution might permit an easy and rapid method to quantify the stress distribution in clinical settings. However, only one experimental test has been performed so far [70]: further research efforts are thus required in this field.

\section{Solutions to optimize interfacial stresses}

Some specific solutions aiming at reducing stress-related skin problems have been proposed in the state of the art. For example, the use of liners allows a cushioned protection for bony prominences [45], as already discussed. It has been also shown that the pressure is better distributed and tolerated when subatmospheric suspensions are used [42]. These systems (liners and subatmospheric suspensions) also guarantee an increase of friction and thus better adhesion to the skin [3].

Variable stiffness sockets have been recently proposed in order to actively modulate stress distribution on the tissues. This approach aims to reduce the stress in specific sensitive areas and to guarantee the structural integrity of the socket, thus avoiding to overcome the material mechanical resistance limit [71]. A variable-impedance socket, featured by higher stiffness values in correspondence to softer limb areas and vice versa, was proposed by Sengeh et al. [72]. The authors used a CAD-CAM process based on laser scanning and MRI data of tissue distribution to design a heterogeneous socket, which decreased contact pressures on bony prominences by 7 $21 \%$, depending on the anatomic area, respect to a bare carbon fiber socket with the same design. Nehme et al. [73] proposed another socket solution based on heterogeneous materials and openings. However, it was validated only with FEA and no tests on amputees have been carried out, yet.

A smart solution to control both stiffness and volume was based on magnetorheological fluids (Fig. 9) [57]. In this case, the volume of a MR bag iwas adjusted through a cylinder. Then, by playing with magnet configurations (magnets were manually positioned in the outside socket wall), it was possible to regulate the overall stiffness. Using a $0.38 \mathrm{~T}$ magnet at the patella area, the pressures decreased from 170 $\mathrm{kPa}$ to $70 \mathrm{kPa}$, in comparison with a TSB socket. However, the mechanical control circuit and the power supply required for the regulation mechanism were rather bulky.
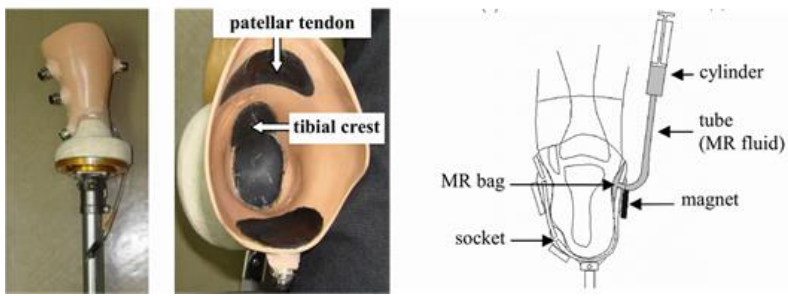

Fig. 9. Variable-stiffness prosthetic socket based on a magnetorheological fluid (reproduced with permission from IEEE [57]).

A reliable and continuous measurement of stresses during daily activities would allow to drive a socket adaptation mechanism, thus to keep stresses in a certain "safety range" and thus to reduce skin problems [74]. Recently, stress sensors have been integrated into the socket wall (as done by [75] and [76]) or in the liner (as done by [62], [60], [61] and proposed by [77]). At the moment, only few closed-loop adaptable systems are available in the literature. A transtibial one was proposed by Pirouzi et al. (Fig. 10) [74], based on an air cuff, regulated by a microcontroller thanks to the data derived from a semiconductor pressure sensor. 
The pressure threshold was set a priori by the user and the volume of the air cuff increased until the set value was reached. In this case, tests with five patients showed smaller mean peak pressure values, in comparison with other studies focusing on sockets coupled with Dermo and Seal-In liners.

In some cases, such as the Hi-Fi socket described above (Fig. 4), high pressure values can be voluntarily applied to fully compress the residual limb, stabilize the bone and reduce the mobility of soft tissues [15], [78]. Alley et al. [15] considered a typical soft tissue stress-strain curve and supposed that a pressure between 2 and $6 \mathrm{MPa}$ could be constantly applied during the day without any discomfort, in order to achieve a good stabilization. A proper study of tissue perfusion in compressed and relaxed areas is still necessary, to avoid a possible ischemic risk associated with such an approach.

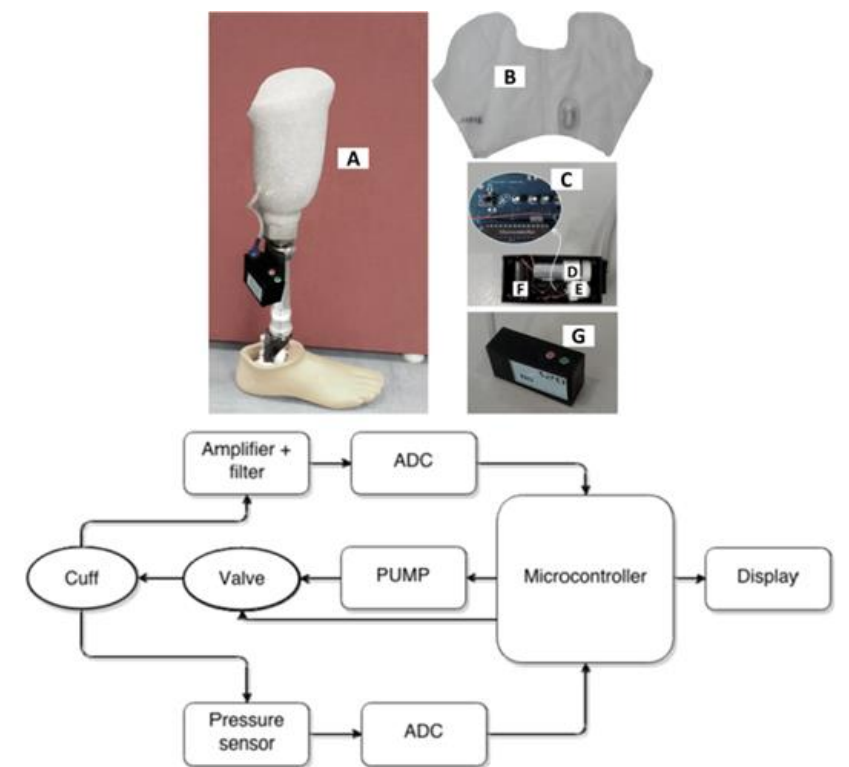

Fig. 10. The Pirouzi's dynamic socket for transtibial amputees (b) (prosthesis (A), bladder (B), control board (C), pump (D), valve (E); Battery (F), and operation system $(\mathrm{G})$, reproduced with permission from MDPI [74]).

\section{Open challenges and future routes}

As already discussed, interfacial stresses are the main cause of skin problems and pain, greatly influencing the overall patient comfort. One of the main challenges is to identify stress threshold values, which should be not overcame, during the prosthesis daily use. To this purpose, further efforts must be devoted to evaluate the stress distribution for different amputation levels and different socket types. This would bring important benefits to research efforts on FEA models, which could compare their predictions with reliable and statistically significant data. FEA simulations are designed based on the stump tissue distribution and aim at identifying possible critical points in which stress may accumulate during dynamic tasks. Reliable data derived from these analysis would allow the design of heterogeneous multi-material sockets whose mechanical properties are optimized, taking into account such critical conditions.

Limitations also concern a possible hardware to be used to measure both normal and shear stresses, without interfering with the socket biomechanics and comfort. Indeed, the few sensors commercially available at present, only measure pressures. An efficient sensor measuring both pressures and shear stresses with low damage risks and without hampering patient comfort still has to be devised. On the other hand, ANN methods might be the most promising solution to overcome these issues, if the predicted results will be successfully validated in a significant number of patients.

To reduce or to better distribute the stresses at the stumpsocket interface, several approaches could be adopted. Among them, the use of heterogeneous materials, featured by different mechanical properties and stiffness gradients with a mm-scale or even greater resolution, seems a promising solution. To this aim, modern additive manufacturing technologies [79], [80] could be evolved and combined with novel materials formulation, in order to achieve this objective.

Closed-loop systems adapting their internal shape and features in order to achieve a better stress distribution are also promising. At present, only few papers followed this route and the resulting systems appeared quite bulky and not much userfriendly. An alternative technology, able to solve both the issue of high interfacial stresses and volume fluctuations (see next section), could be based on granular jamming. This approach relies on elastic chambers filled with granular structures [81]. When vacuum is applied into the chambers, the system stiffness increases. This may guarantee higher stiffness in case of limb volume increase, as usually occurs at the softer tissue regions. No studies following this approach have been pursued in literature, so far.

The development of complex sockets, provided with active stiffness-regulating mechanisms and a closed-loop control based on embedded sensors, looks promising, also because they may address further issues (temperature, volume, etc.) by using the same technologies used to optimize interfacial stresses, or slightly adapting them.

\section{VOLUME FLUCTUATIONS}

\section{A. Volume fluctuations: general considerations}

One of the main goals of prosthetic sockets is to achieve an optimal fit. However, this requires to develop a system able to compensate changes in volume and shape [82]. Indeed, stump volume losses cause displacements and enhance the pistoning effect. On the other hand, if the stump volume increases, high pressures and shear stresses arise at the skin [83]. These changes are critical, especially in the post-amputation phase, featured by edema and muscle atrophy, and continue to be visible also in mature stumps (after 12-18 months), worsened by vascular diseases or other co-morbidities [10], [43]. Different measurement techniques exist to evaluate volume changes: water displacement techniques, directly applied on the stump or on a cast, simple anthropometric measurements made through tapes or calipers, optical scanning, contact probes, ultrasound, spiral X-ray computer tomography, laser scanning, MRI, and bio-impedance [43].

So far, volume change due to muscle contraction has been investigated only on transtibial amputees. It was quantified in 
$+5.8 \%$ in absence of a liner and in $+3.5 \%$ when a liner was used [84]. Volume changes due to liquid movements can occur in the short term (hours, days) or in the long term (weeks, months, years). In general, the stump is subjected to daily volume fluctuations which range from $-11 \%$ to $+7 \%$ or even more [83], [85].

Other studies suggested that a volume increase of $3-5 \%$ is sufficient to cause discomfort and difficulties in donning [83]. In lower limb amputees the speed of volume changes can be around $0.10-0.12 \mathrm{~mL} / \mathrm{min}$ during standing and $0.20-0.30$ $\mathrm{mL} / \mathrm{min}$ after motion [86]. The variation rate also depends on the suspension type and on the socket size. Board et al. observed that vacuum-assisted suction $(-78 \mathrm{kPa})$ induced a volume gain of $3.7 \%$ against a loss of $6.5 \%$ without vacuum suspension [83]. This study was carried out on TSB sockets and after 30 min of treadmill walking. In general, most studies showed a gain in volume when negative pressures were applied within the socket. Conversely, non-VAS suspensions mainly cause volume losses [42], [83]. Comparing undersized $(-15 \%)$, natural sized $(-7 \%)$, and oversized $(+3 \%)$ transtibial sockets, a loss of $2 \%$, a gain of $5 \%$ and a gain of $11 \%$ in volume have been registered, respectively, after $18 \mathrm{~min}$ of walking [6], [87]. All the mentioned values have been measured mainly on transtibial amputees. Thus, studies focusing on the other amputation levels are still needed.

\section{B. Technologies to compensate volume fluctuations}

Different methods have been suggested for facing daily volume fluctuations. The use of flexible socket systems has been proposed to this purpose. They can consist of a polyethylene or silicone elastomer inner socket with an outer harder structure, provided with fenestrations. Some solutions for lower limbs are the IPOS, the ISNY (Icelandic Swedish New York) and the SFS (Scandinavian Flexible Socket), which differ in terms of frame fabrication techniques and inner socket materials [88].

Other solutions include socks, pads or inflatable bladders, which can be added or integrated into the socket (Fig. 11a). Sanders et al. reviewed the mechanical features of commercially available air-filled bladders [89]. The main issues affecting them are a high compressibility, which causes losses [43], and the need of high pressures to reduce the volume variability [90]. Furthermore, they are more difficult to control in comparison with liquid actuation systems [91].

Another solution proposed for lower limb was based on an array of sensorized inflatable pressure actuators, inserted in special pockets within the socket wall [92] (Fig. 11b). In this first prototype an external pump, connected with an air pressure regulator, was used to actuate the inflatable array, while an F-Socket sensor read the pressures at the interface. Future evolutions of this system will likely aim at miniaturizing the setup, thus to efficiently integrate all the components within a socket. A similar solution was based on an all-covering bladder with a controller (Fig. 10) [74].

However, as previously discussed, liquid-filled solutions would be preferable. The only commercial hydraulic circuit integrated inside a socket is described in [90] (Fig. 11c). is the authors described a passive system in which the fluid was drawn from a reservoir into some bladders by exploiting leg motion and by gravity, whereas unidirectional valves avoided a reflux. Another fluidic solution is the one based on magnetorheological fluids described in the previous section and shown in Fig. 9 [57]. Magnetorheological fluids have the advantage of allowing a double control on volume and stiffness. However this solution is limited by its bulkiness, as already discussed. A fluidic solution can be also obtained by localizing rolled fluidic artificial muscles in sensitive areas within the socket. In these actuators, inextensible braided fibers transform a balloon isotropic strain into a contraction or an extension, allowing a uniform strain and supporting high loads in comparison to simple bladders [86].

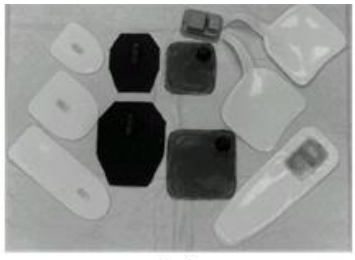

(a)

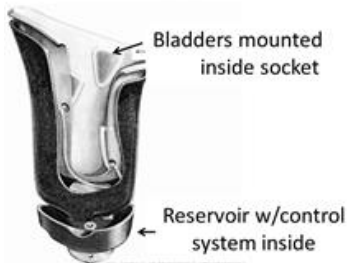

(c)

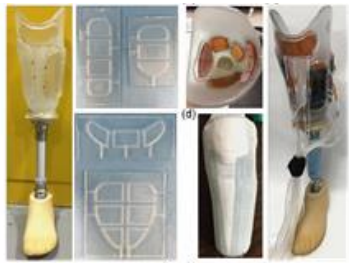

(b)
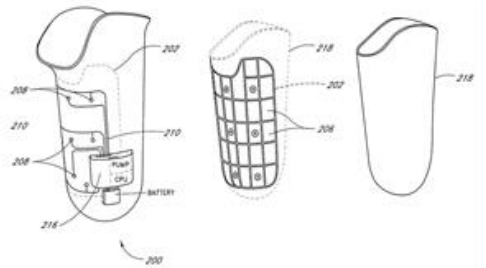

(d)
Fig. 11. Some examples of inflatable inserts for prosthetic socket (a) and of sockets with integrated bladders (b-d) (a reproduced with permission from U.S. Department of Veterans Affair [89], b reproduced with permission from IEEE [92], c reproduced with permission from American Academy of Orthotists and Prosthetists [90], d adapted from [93])

A patent analysis in this domain revealed other interesting solutions. Some of them are related to insertable bladders, manually inflated with air by the prosthetist [94] or through an external needle [95]. Another solution consists of an insert made of several bladders, integrated within the inner socket wall and actively controlled by a central processing unit (CPU), closing the loop with pressure sensors (Fig. 11d) [93]. A similar concept has been applied to the liner [96]. The system proposed in this patent is composed of two layers made of a porous matrix and an incompressible fluid, which can flow from the outer to the inner layer, under the control of a CPU, by using the output of pressure sensors. In order to compensate volume fluctuations, the liner could be also made of an auxetic foam, which expands or reducesas the limb volume decreases or increases [97].

Other approaches exist, based on sockets made of movable panels (Fig. 12). These can be adjusted manually through straps [98] [99], clamps (Fig. 12a) [100] [101] or lacing system (e.g. the commercial RevoFit socket with the Boa system). In other configurations, the socket can be divided in struts, connected through encircling bands (Fig. 12b) [102] or through a lacing system with a tensioning mechanism (Fig. 12c) [103], [104], which is manually adjustable. 


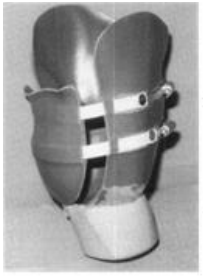

(a)

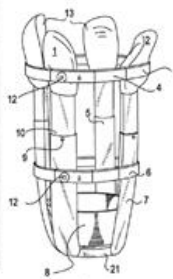

(b)

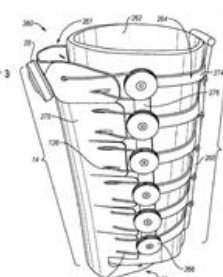

(c)
Fig. 12. Examples of variable-volume sockets (a reproduced with permission from American Academy of Orthotists and Prosthetists [100], b adapted from [102], and c adapted from [103]).

The most recently launched commercial solutions are based on this endeavor. The Infinite Socket ${ }^{\mathrm{TM}}$ (LIM Innovations, San Francisco, CA, USA) is an innovative commercial custom socket based on four struts, which can be adapted to the stump volume thanks to pivoting and sliding joints, lacing system and clamps, thus improving the user performances, as demonstrated in [105] (Fig. 13). Another efficient solution is the Socket-less Socket ${ }^{\mathrm{TM}}$ (Martin Bionics, Oklahoma City, USA), able to perform macro and micro-adjustments on volume fluctuations, but no studies on this design have been found in the literature. Both systems are manually adjustabe by the patient. However, to avoid an excessive tightening, which may have severe consequences in the long term (stump deformation and mass loss), these devices should evolve towards sockets able to automatically adjust their volume.

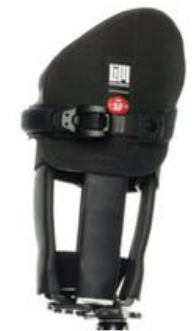

(a)

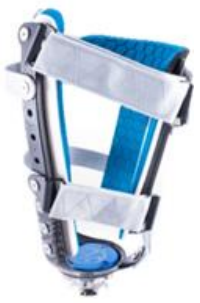

(b)
Fig. 13. The Infinite Socket ${ }^{\mathrm{TM}}$ (a, photo courtesy George Burnard/ LIM Innovations, www.liminnovations.com) and the Socket-less Socket ${ }^{\mathrm{TM}}$ (b, photo courtesy Jay Martin/ Martin Bionics, www.martinbionics.com).

\section{Open challenges and future routes}

Regarding volume adjustments, several solutions have been proposed but a generally accepted solution is not available, yet. The use of bladders, pads or other inserts does not allow a uniform deformation across the socket thickness and, consequently, does not guarantee a uniform stress distribution [43], [86]. In addition, the use of inserts within the socket has to be more carefully investigated. In fact, Sanders et al. claimed that such elements may induce an additional volume loss, due to non-physiological stump tissue compression [82]. If the Sanders' assumption will be not confirmed, a smart design based on fluidic chambers could be an efficient solution to be pursued, in order to better distribute the stresses and properly adjust the volumes, when the stump volume tends to decrease. In addition, through magnetorheological fluids, also the socket wall stiffness could be regulated. Contrarily, if the stump volume tends to increase, the granular jamming technology, previously described, could be adopted. Since volume gains occur especially with VAS suspension and considering that the granular jamming concept is based on the application of vacuum inside elastic chambers, a single vacuum pump could be used for both purposes: VAS and jamming.

Current commercial solutions are based on lacing or clamp systems manually adjustable by the users: none of them adjust the volume automatically. This may bring patients to exceed in tightening their socket, driven by the desire of maximizing the fitting and stability of their prosthesis. An excessive tightening, however, is likely to cause dramatic volume changes in the residual tissues and dermatological problems, thus increasing the risk of prosthesis abandon, in the longterm. In general, a dynamic system able to monitor the stress distribution and consequently adjust the volume and the shape of the socket would be much more efficient. Future efforts may be focused in this direction.

\section{TEMPERATURE}

\section{A. Thermoregulation and comfort: a close relationship}

Thermal homeostasis within the socket is another key factor for the long-term success of prostheses. Previous studies have found that more than $53 \%$ of prosthetic users feel discomfort due to excessive heat or sweating, and an increment of $1-2^{\circ} \mathrm{C}$ is sufficient to trigger this kind of problems [106].

After amputation, the thermoregulatory system tends to increase the patient's sweating rate since full-contact sockets with liners, which represent a good solution in terms of fitting, create a barrier to thermal transfer mechanisms [107], [108].

Materials that are nowadays widespread in prosthetic sockets and liners (i.e., silicone, thermoplastic elastomers, mineral oil gel, closed cell foam, etc.) are featured by poor heat conduction capabilities (thermal conductivity lower than $1 \mathrm{~W} / \mathrm{m} \cdot \mathrm{K})$ and low moisture permeability [109], [110]. Consequently, skin maceration and bacterial invasion may occur [111]. The typical amputee clinical picture worsens this situation: such patients are often affected by diabetes or vascular diseases, with sweating abnormalities and a reduced convective mechanism by the circulatory system [10]. Thus, proper materials have to be devised for sockets, taking into account their thermal conductivity and breathability in addition to their friction coefficient and overall elasticity.

Individual aspects, such as age, sex and activity level are also significant in the thermoregulatory process [112]. This makes it difficult to obtain a temperature absolute value which has to be maintained at the skin level of prosthetic users. However, average values of skin temperature are around $31^{\circ}$, which can be used as a typical reference value for achieving thermal comfort [113], [114]. In general, the skin temperature is lower in areas featured by low perfusion, such as the anterior proximal locations, and higher in areas with high perfusion, e.g. across the posterior section. Close to large muscle masses, metabolism and perfusion are greater than close to bony regions. This is reflected by corresponding higher and lower skin temperatures, respectively [115], [116].

Quantifying temperature fluctuations at the stump-socket interface is an important starting point in order to identify 
suitable technological solutions. Perry et al. analyzed skin temperature within the socket of 5 transtibial amputees during different activities, by using ad hoc thermistors [115]. The temperature, which had a mean initial value of $31.4^{\circ}$, increased by $0.8^{\circ}$ when the patient remained in a seated position for $15 \mathrm{~min}$, and by $1.7^{\circ}$ when the patient walked for 10 min. After improving the experimental set up [116], a new analysis on a transtibial amputee was performed. The skin temperature decreased by $0.4^{\circ}$, reaching $29.5^{\circ}$, when the patient rested for one hour, whereas it increased by $3.1^{\circ}$ after walking for one hour.

\section{B. Existing solutions to avoid stump overheating}

Several solutions to avoid the discomfort caused by heat and perspiration have been proposed, although only few of them are already available. Some possible solutions focused on the properties of certain materials, e.g. breathable fabrics [106]. The Silcare Breathe Liner by Endolite (Basingstoke, Hampshire, UK) is a commercial solution in which air and sweat, normally trapped between the liner and the skin, are expelled thanks to laser-drilled perforations on the liner, thus guaranteeing a healthier environment for the stump [117].

Textile spacer fabrics with bacteriostatic fibers and silver ions can be also added to liners to prevent bacterial invasion and to reduce unpleasant odors; in addition, they allow good capillary effects eliminating perspiration [109]. In this framework, available commercial solutions are socks based on the X-Static ${ }^{\circledR}$ technology (based on pure silver-coated fibers), which have to be worn below the liner, allowing antimicrobial and anti-odor benefits.

Nanocomposite materials may constitute an added value in this field. Nanotechnology-based solutions can be exploited to increase hygiene, by fabricating super-hydrophobic surfaces or by adding silver antibacterial particles, to reduce sweat and odor build-up produced at the socket interface [106], [118]. Graphitic foam materials may be also adopted, to achieve products with higher thermal conductive properties.

Cooling vests can be also obtained by using smart materials which can absorb or release heat, according to their change phases [118]. An innovative commercial solution exploiting this paradigm is the SmartTemp ${ }^{\circledR}$ Liner by The Ohio Willow Wood Company (Mt Sterling, OH, USA) [117]. It is based on the NASA Outlast ${ }^{\circledR}$ technology, incorporating phase change materials in the silicone liner. When the skin is exposed to temperature increments, the expelled heat is absorbed by the liner and released when the skin temperature decreases. By testing this liner on 16 transtibial amputees, Wernke et al. have demonstrated a significant reduction in skin temperature and sweating during activity and post-activity [119].

An alternative approach consists of developing a socket with ventilated shells, through some openings parts which allow the escape of moisture and increase skin ventilation [120]. However, they enhance stresses at the brims and reduce only partially the thermal discomfort, thus not representing a game-changing solution.

More complex systems, based on active thermoregulation devices or phase change materials are promising, although they should be miniaturized and more properly controlled in order to be integrated in commercial devices. A proposed solution is based on the phase change of an ice pack, used as a dedicated element to absorb heat in a cooling system integrated into the socket wall (Fig. 14a) [121]. Using a preliminary prototype, its cooling capability resulted from 6.6 $\mathrm{W}$ to $15.6 \mathrm{~W}$ thanks to the adjustable thermal resistance obtained through the flow channel array.

Another thermoregulated socket was described in [114], [122] (Fig. 14b). This device received data from 16 different integrated digital temperature sensors, sent such inputs to a microcontroller and compared them with a standard temperature value, previously set. The result of this comparison was then used to activate a cooling/heating system, based on a thermal pump. Through bench tests, the feasibility of this approach has been confirmed: the device was able to guarantee thermal equilibrium between the outside and the inside of the liner. However, further investigation and validation steps are needed.

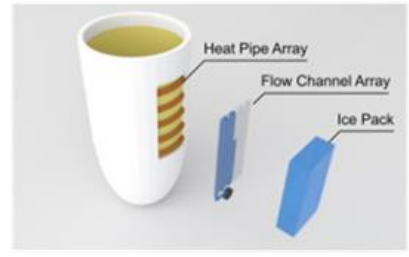

(a)

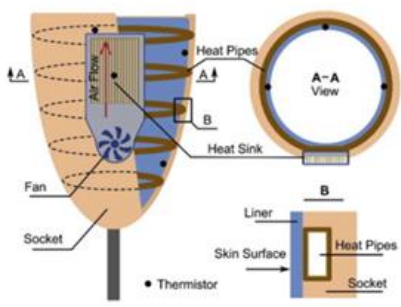

(c)

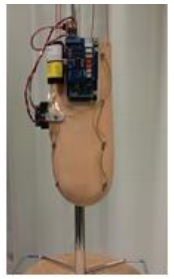

(b)

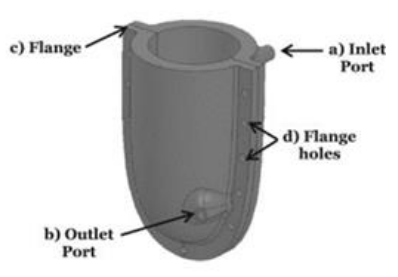

(d)
Fig. 14. Thermoregulatory systems within prosthetic sockets based on the phase change of an ice pack (a), on a closed-loop system with a thermal pump (b), on a heat sink and a fan (c) and on a cooling channel with an air pump (d) (a, c and d reproduced with permission from Elsevier [121], [123], [124], b photo courtesy Kamiar Ghoseiri/ Department of Orthotics and Prosthetics, University of Social Welfare and Rehabilitation Sciences).

Another proposed design was based on heat pipes with a negligible thermal resistance, which concentrated the heat flux from the liner to a heat sink. A compact fan then moved the heat flux from the heat sink to the surrounding environment (Fig. 14c) [123]. The preliminary prototype showed a cooling capability ranging from $2.1 \mathrm{~W}$ to $7.0 \mathrm{~W}$ and it was able to maintain a constant temperature. Other groups also tried to modify the socket wall, incorporating a helical cooling channel inside it, with an air pump embedded at the inlet, in order to circulate room temperature air within the socket. They demonstrated a cooling effect through computer simulations and bench tests (Fig. 14d) [124].

Among the sockets with ventilated shells, there are examples of patented prostheses with a socket wall divided into different parts, connected through straps or similar elements and comprising a ventilated spacer element in the breathable fabric (Fig. 15a) [125]. Another patented solution close to this concept is based on a reconfigurable socket with a 
window and a panel, adjustable by a lacing system [126]. It also comprises a membrane, permeable to water but not to the air, and a porous layer, which allows the elimination of moisture.

Further strategies have been recently patented. One of them is based on thermoelectric coolers (TEC), based on the Peltier effect, embedded into the socket wall [127] or into the liner [97], offering rather compact, yet controllable solutions (Fig. 15b). TEC devices are featured by low cooling performances, due to their limited heat exchange efficiency, and by a rather high power consumption. However some improvements have been recently achieved for this technology [128].

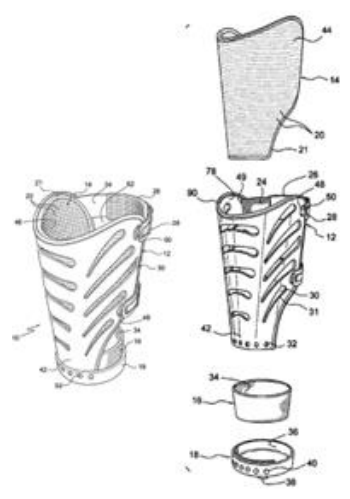

(a)

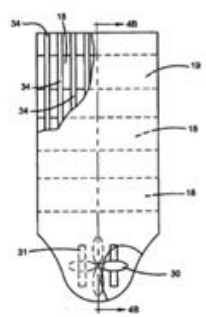

(c)

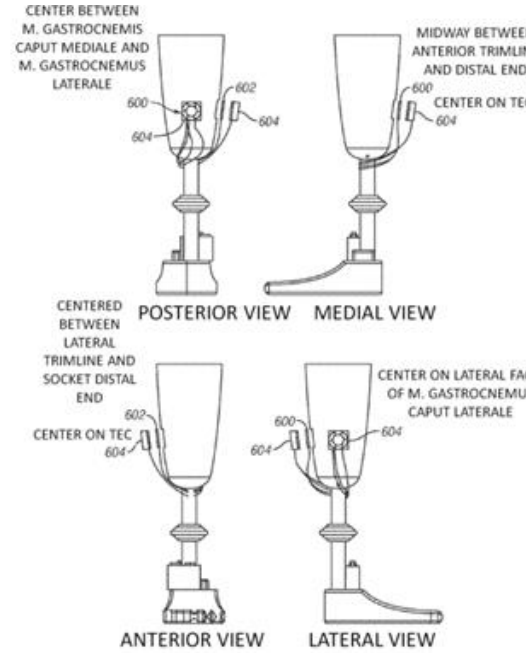

(b)

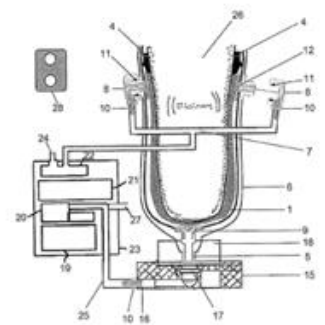

(d)
Fig. 15. Patented thermoregulation systems for sockets, based on ventilated spacer element and breathable fabric (a), openings in the socket wall (b), a closed-loop cooling system with a heat pipe and a heat sink (c) and a liner with airflow channels (d) (a adapted from [125], b adapted from [127], c adapted from [129], $\mathrm{d}$ adapted from [130]).

Zhe et al. [129] proposed an integrated cooling system which comprises a heat pipe to focus the heat flux through a working fluid to a heat sink, regulated by a control system and heat sensors (Fig. 15c). Fluid evaporation draws heat, and subsequently the heat sink decreases its temperature. The device includes a vacuum system in the heat pipe for changing the fluid boiling point with respect to the boiling point at atmospheric conditions, and a fan for blowing the air across the heat pipe. Another patent [131] proposes a device which concentrates air in a canalized layer, allowing the absorption of moisture and the evaporation of perspiration. Near the canalized layer there is another layer with highly conductive fibers that redistribute heat uniformly. In [104] a porous wicking material, attached to a hypobaric assisted vacuum liner, is suggested to allow moisture escapement. Finally, in [130] a dedicated liner with different conical holes is proposed; the holes would be able to eliminate moisture at the skin through airflow channels used also for the suspension (Fig. 15d).

\section{Open challenges and future routes}

Rather few studies have been dedicated to temperature measurements on the residual limb. Thus, a better characterization is still necessary, especially concerning transfemoral amputees. A main obstacle is the use of thermistors, which allow to carry out only short tests, because they cause discomfort, irritations and skin ulcerations. Ad hoc measurement systems should be developed to this purpose. Possible solutions could be based on optical fibers developed through micro electro-mechanical systems (MEMS) technologies and able to measure both temperature and pressure values [132]. MEMS sensors could be used also to evaluate the moisture within a socket [133] and in general may allow multi-purpose and multi-variable sensing [134].

Despite several solutions have been devised to avoid stump overheating, only two dedicated liners are commercially available (The Silcare Breathe Liner by Endolite and the SmartTemp ${ }^{\circledR}$ Liner by The Ohio Willow Wood Company). Future efforts may be focused on improving material proprieties, to make them more hygienic and breathable.

Nanotechnology may play an important role in finding efficient solutions for heat dissipation. However, nanomaterials safety is still a debated issue: if the material degrades over time, nanoparticles could be released on the skin, thus causing irritations, allergic reactions or even worst effects [106], [135]. More studies are thus required to investigate their stability and their possible toxicity.

Phase change materials are a very interesting alternative, although they are quite hard to control. For such solutions, as well as for complex thermoregulation mechatronic systems, it must be taken into account that local skin cooling can generate a localized vasoconstriction, which decreases skin blood flow almost to zero. Thus, a more accurate thermodynamic investigation has to be performed to evaluate the use of such technologies in the field of prosthetic sockets, besides the technical problems related to miniaturization and usability.

\section{CONCLUSION}

The field of prosthetics dramatically evolved in the last decades. However, many amputees still reject their prostheses or report a low satisfaction level, mainly due to socket-related issues. An interesting approach that tries to overcome this limitation is based on osseointegration [158]. In this paradigm, a fixed component is implanted into the bone and a percutaneous abutment connects it to the prosthesis. This approach allows recovering the physiological load transmission through the skeleton. However, several risks arise at the interface, often causing re-interventions, fractures, etc. Hence, it is still far from fully substituting prosthetic sockets. 
The development of an ideal socket is a non-straightforward process, which needs to address a complex interplay of factors affecting the long-term usability, comfort and overall performance of the prosthesis. Such factors can be separately identified and described, but they all act in a synergistic way to determine the socket success or failure. Interfacial stresses are one of the most important factors to be considered. An altered stress distribution can cause skin problems and pain, affecting the whole comfort and, consequently, the gait biomechanics. The stump volume fluctuations alter the socket fitting, donning and comfort. Temperature is another important factor: an increase of temperature can lead to perspiration, which increases slippage and causes skin problems. Slippage obviously alters the socket fit, with consequences also on the stress distribution.

To date, the socket design is mainly grounded on the prosthetist's experience, with poor attention to quantitative data. Associating the invaluable know-how of prosthetists with advanced materials, miniaturized and - as much as possible - "disappearing" sensors and actuators will be probably the right way to achieve a really advanced socket, able to self-adapt to interfacial stresses, volume and temperature fluctuations in real-time and in a closed-loop fashion, during both static and dynamic tasks.

\section{REFERENCES}

[1] M. Marino et al., "Access to prosthetic devices in developing countries: Pathways and challenges," in Proc. IEEE Annu. Conf. GHTC, 2015, pp. 45-51.

[2] K. Ziegler-Graham et al., "Estimating the Prevalence of Limb Loss in the United States: 2005 to 2050," Arch. Phys. Med. Rehabil., vol. 89, no. 3, pp. 422-429, Mar. 2008.

[3] A. F. Mak et al., "State-of-the-art research in lower-limb prosthetic biomechanics-socket interface: a review," JRRD, vol. 38, no. 2, pp. 161-74, Apr. 2001.

[4] T. R. Dillingham et al., "Use and satisfaction with prosthetic devices among persons with trauma-related amputations: a long-term outcome study," Am. J. Phys. Med. Rehabil., vol. 80, no. 8, pp. 56371, Aug. 2001.

[5] H. E. Meulenbelt et al., "Determinants of Skin Problems of the Stump in Lower-Limb Amputees," Arch. Phys. Med. Rehabil., vol. 90, no. 1, pp. 74-81, Jan. 2009.

[6] M. R. Safari and M. R. Meier, "Systematic review of effects of current transtibial prosthetic socket designs-Part 2: Quantitative outcomes," JRRD, vol. 52, no. 5, pp. 509-526, Jan. 2015.

[7] E. A. Al-Fakih et al., "Techniques for interface stress measurements within prosthetic sockets of transtibial amputees: A review of the past 50 years of research," Sensors, vol. 16, no. 7, pp. 1119, Jul. 2016.

[8] S. Chatterjee et al., "Review: Problems with Use of Trans-Tibial Prosthesis," J. Med. Imaging Heal. Informatics, vol. 6, no. 2, pp. 269-284, Apr. 2016.

[9] J. A. De Lisa et al., Physical Medicine and Rehabilitation: Principles and Practice. Hagerstown, MD: Lippincott Williams \& Wilkins, 2005.

[10] J. T. Highsmith and M. J. Highsmith, "Common skin pathology in LE prosthesis users: the nature of state-of-the-art skin-prosthesis interface puts amputees who use prostheses at increased risk for these common dermatologic conditions," JAAPA, vol. 20, no. 11, pp. 33-38, Nov. 2007.

[11] I. Long, "Allowing normal adduction of femur in above- knee amputations. Technical note," Orthot. Prosthet., vol. 29, no. 4, pp. 53-54, 1975.

[12] G. Rubin et al., "Prescription of above-knee and below-knee prostheses," Prosthet. Orthot. Int, vol. 10, no. 3, pp. 117-24, Dec. 1986.

[13] J. T. Andrew, "Transhumeral and Elbow Disarticulation Anatomically Contoured Socket Considerations," JPO, vol. 20, no.
3, pp. 107-117, Jul. 2008.

[14] S. Fatone and R. Caldwell, "Northwestern University Flexible Subischial Vacuum Socket for persons with transfemoral amputation: Part 2 Description and Preliminary evaluation," Prosthet. Orthot. Int, vol. 41, no. 3, pp. 246-250, Jun. 2017.

[15] R. D. Alley et al., "Prosthetic sockets stabilized by alternating areas of tissue compression and release," JRRD, vol. 48, no. 6, pp. 67996, Jun. 2011.

[16] W. C. Miller et al., "The prevalence and risk factors of falling and fear of falling among lower extremity amputees," Arch. Phys. Med. Rehabil., vol. 82, no. 8, pp. 1031-1037, Aug. 2001.

[17] J. M. Czerniecki, "Rehabilitation in limb deficiency. 1. Gait and motion analysis," Arch. Phys. Med. Rehabil., vol. 77, no. 3, pp. S3S8, Mar. 1996.

[18] M. Montero-Odasso et al., "Gait Velocity as a Single Predictor of Adverse Events in Healthy Seniors Aged 75 Years and Older," $J$ Gerontol. Ser. A: Biol. Sci. Med. Sci., vol. 60, no. 10, pp. 13041309, Oct. 2005.

[19] C. W. Radcliffe, "Functional considerations in the fitting of aboveknee prostheses," Artif. Limbs, vol. 2, no. 1, pp. 35-60, Jan. 1955.

[20] F. Flandry et al, "The effect of the CAT-CAM above-knee prosthesis on functional rehabilitation," Clin. Orthop. Relat. Res., vol. 239, pp. 249-62, Feb. 1989.

[21] R. S. Gailey et al., "The CAT-CAM socket and quadrilateral socket: a comparison of energy cost during ambulation," Prosthet. Orthot. Int., vol. 17, pp. 95-100, Aug. 1993.

[22] J. T. Kahle and M. J. Highsmith, "Transfemoral interfaces with vacuum assisted suspension comparison of gait, balance, and subjective analysis: Ischial containment versus brimless," Gait Posture, vol. 40, no. 2, pp. 315-320, Jun. 2014.

[23] J. T. Kahle et al., "The Effect of Transfemoral Interface Design On Gait Speed and Risk of Falls," Technol. Innov., vol. 18, no. 2, pp. 167-173, Sep. 2016.

[24] R. Alley, "The High-Fidelity Interface : Skeletal Stabilization through Alternating Soft Tissue Compression and Release," in Proc. MyoElectric Controls/Powered Prosthetics Symposium Fredericton, 2011, pp. 3-5.

[25] T T. Schmalz et al., "Energy expenditure and biomechanical characteristics of lower limb amputee gait: The influence of prosthetic alignment and different prosthetic components," Gait Posture, vol. 16, no. 3, pp. 255-263, Dec. 2002.

[26] R. Versluys et al., "Prosthetic feet: State-of-the-art review and the importance of mimicking human ankle-foot biomechanics," Disabil. Rehabil. Assist. Technol., vol. 4, no. 2, pp. 65-75, Jan. 2009.

[27] M. D. Muller, "Transfemoral Amputation: Prosthetic Management," in Atlas of of amputation and limb deficiencies, $4^{\text {th }}$ ed., Rosemont, IL, USA: Amer. Acad. Orthopaedic Surgeons, 2016, pp. 537-554

[28] J. Gardiner et al., "Transtibial amputee gait efficiency: Energy storage and return versus solid ankle cushioned heel prosthetic feet," JRRD., vol. 53, no. 6, pp. 1133-1138, 2016.

[29] H. M. Herr and A. M. Grabowski, "Bionic ankle-foot prosthesis normalizes walking gait for persons with leg amputation," in Proc. R. Soc. B, vol. 279, no. 1728, 2011.

[30] M. Windrich et al., "Active lower limb prosthetics: a systematic review of design issues and solutions," Biomed. Eng. Online, vol. 15, no. S3, pp. 140, Dec. 2016.

[31] R. Jiménez-Fabián and O. Verlinden, "Review of control algorithms for robotic ankle systems in lower-limb orthoses, prostheses, and exoskeletons," Med. Eng. Phys., vol. 34, no. 4, pp. 397-408, May 2012.

[32] P. K. Kumar et al., "Trends and Challenges in Lower Limb Prosthesis," IEEE Potentials, vol. 36, no. 1, pp. 19-23, Jan. 2017.

[33] G. K. Klute et al., "Mechanical properties of prosthetic limbs: adapting to the patient.," JRRD, vol. 38, no. 3, pp. 299-307, 2001.

[34] C. Pew and G. K. Klute, "Pilot testing of a variable stiffness transverse plane adapter for lower limb amputees," Gait Posture, vol. 51, pp. 104-108, Jan. 2017.

[35] E. S. Neumann, "State-of-the-Science Review of Transtibial Prosthesis Alignment Perturbation," JPO, vol. 21, no. 4, pp. 175193, Oct. 2009.

[36] P. Davenport et al., "Systematic Review of Studies Examining Transtibial Prosthetic Socket Pressures with Changes in Device Alignment," J. Med. Biol. Eng., vol. 37, no. 1, pp. 1-17, Feb. 2017.

[37] S. R. Koehler-McNicholas et al., "The biomechanical response of 
persons with transfemoral amputation to variations in prosthetic knee alignment during level walking," JRRD, vol. 53, no. 6, pp. $1089-1106,2016$.

[38] G. K. Klute et al., "Prosthetic Liners for Lower Limb Amputees: A Review of the Literature," Prosthet. Orthot. Int, vol. 34, no. 2, pp. 146-153, Jun. 2010.

[39] H. Gholizadeh et al., "Satisfaction and Problems Experienced With Transfemoral Suspension Systems: A Comparison Between Common Suction Socket and Seal-In Liner," Arch. Phys. Med. Rehabil., vol. 94, no. 8, pp. 1584-1589, Aug. 2013.

[40] H. Gholizadeh et al., "The evidence-base for elevated vacuum in lower limb prosthetics: Literature review and professional feedback," Clinical Biomechanics, vol. 37, pp. 108-116, 2016.

[41] J. T. Kahle and M. J. Highsmith, "Transfemoral sockets with vacuum-assisted suspension comparison of hip kinematics, socket position, contact pressure, and preference: ischial containment versus brimless.," JRRD, vol. 50, no. 9, pp. 1241-52, 2013.

[42] J. T. Kahle et al., "The Effects Of Vacuum-Assisted Suspension On Residual Limb Physiology, Wound Healing, And Function: A Systematic Review," Technol. Innov., vol. 15, no. 4, pp. 333-341, Jan. 2014.

[43] J. E. Sanders and S. Fatone, "Residual limb volume change: systematic review of measurement and management," JRRD, vol. 48, no. 8, pp. 949-86, 2011

[44] A. Eshraghi et al., "Pistoning assessment in lower limb prosthetic sockets," Prosthet. Orthot. Int, vol. 36, no. 1, pp. 15-24, Mar. 2012.

[45] E. C. T. Baars and J. H. B. Geertzen, "Literature review of the possible advantages of silicon liner socket use in trans-tibial prostheses," Prosthet. Orthot. Int, vol. 29, no. 1, pp. 27-37, Apr. 2005

[46] K. Yiğiter et al., "Comparison of the effects of patellar tendon bearing and total surface bearing sockets on prosthetic fitting and rehabilitation," Prosthet. Orthot. Int, vol. 26, no. 3, pp. 206-212, Dec. 2002.

[47] S. Brunelli et al., "A comparison between the suction suspension system and the hypobaric Iceross Seal-In ${ }^{\circledR}$ X5 in transtibial amputees," Prosthet. Orthot. Int, vol. 37, no. 6, pp. 436-444, Dec. 2013.

[48] A. Eshraghi et al., "Quantitative and Qualitative Comparison of a New Prosthetic Suspension System with Two Existing Suspension Systems for Lower Limb Amputees," Am. J. Phys. Med. Rehabil., vol. 91, no. 12, pp. 1028-1038, Dec. 2012.

[49] G. K. Klute et al., "Vacuum-assisted socket suspension compared with pin suspension for lower extremity amputees: Effect on fit, activity, and limb volume," Arch. Phys. Med. Rehabil., vol. 92, no. 10, pp. 1570-1575, 2011.

[50] H. Gholizadeh et al., "Transtibial prosthetic suspension: less pistoning versus easy donning and doffing.," JRRD, vol. 49, no. 9, pp. 1321-30, 2012.

[51] H. Gholizadeh et al., "Transtibial prosthetic socket pistoning: Static evaluation of Seal-In ${ }^{\circledR}$ X5 and Dermo ${ }^{\circledR}$ Liner using motion analysis system," Clin. Biomech., vol. 27, no. 1, pp. 34-39, Jan. 2012.

[52] P. Convery and K. D. Murray, "Ultrasound study of the motion of the residual femur within a transfemoral socket during gait," Prosthet. Orthot. Int, vol. 24, no. 3, pp. 226-232, Dec. 2000.

[53] J. E. Sanders, "Thermal response of skin to cyclic pressure and pressure with shear: a technical note," JRRD, vol. 37, no. 5, pp. 511-5.

[54] S. Ali et al., "Interface pressure in transtibial socket during ascent and descent on stairs and its effect on patient satisfaction," Clin. Biomech., vol. 28, no. 9-10, pp. 994-999, Nov. 2013.

[55] W. C. Lee et al., "Regional differences in pain threshold and tolerance of the transtibial residual limb: Including the effects of age and interface material," Arch. Phys. Med. Rehabil., vol. 86, no. 4 pp. 641-649, Apr. 2005

[56] G. Colombo et al., "Automatic Below-Knee Prosthesis Socket Design: A Preliminary Approach," in Springer Int. Publ. Switz., 2016, pp. 75-81.

[57] A. Ogawa et al., "Design of lower limb prosthesis with contact pressure adjustment by MR fluid," in 2008 30th Annual Int Conference of the IEEE Eng. in Medicine and Biology Society, 2008, pp. 330-333.

[58] A. Petron et al., "Multi-Indenter Device for in Vivo Biomechanical Tissue Measurement," IEEE Trans. Neural Syst. Rehabil. Eng., vol. 25, no. 5, pp. 426-435, May 2017.
[59] A. S. Dickinson et al., "Finite element analysis of the amputated lower limb: A systematic review and recommendations," Med. Eng. Phys., vol. 43, pp. 1-18, May 2017.

[60] P. Laszczak et al., "Development and validation of a 3D-printed interfacial stress sensor for prosthetic applications," Med. Eng. Phys., vol. 37, no. 1, pp. 132-137, Jan. 2015.

[61] P. Laszczak et al., "A pressure and shear sensor system for stress measurement at lower limb residuum/socket interface," Med. Eng. Phys., vol. 38, no. 7, pp. 695-700, Jul. 2016.

[62] J. Wheeler et al., "A pressure and shear sensing liner for prosthetic sockets," in Proc IEEE Annu. Conf. EMBC, 2016, pp. 2026-2029.

[63] E. A. Al-Fakih et al., "Development and Validation of Fiber Bragg Grating Sensing Pad for Interface Pressure Measurements Within Prosthetic Sockets," IEEE Sens. J., vol. 16, no. 4, pp. 965-974, Feb. 2016.

[64] E. Al-Fakih et al., "The Use of Fiber Bragg Grating Sensors in Biomechanics and Rehabilitation Applications: The State-of-the-Art and Ongoing Research Topics," Sensors, vol. 12, no. 12, pp. 1289012926, Sep. 2012.

[65] D. Tsiokos et al., "Fiber Optic-Based Pressure Sensing Surface for Skin Health Management in Prosthetic and Rehabilitation Interventions," Biomed. Eng. - Tech. Appl. Med., 2012.

[66] P. Sewell et al., "Static and dynamic pressure prediction for prosthetic socket fitting assessment utilising an inverse problem approach," Artif. Intell. Med., vol. 54, no. 1, pp. 29-41, Jan. 2012.

[67] P. Sewell et al., "Improvements in the accuracy of an Inverse Problem Engine's output for the prediction of below-knee prosthetic socket interfacial loads," Eng. Appl. Artif. Intell., vol. 23, no. 6, pp. 1000-1011, Sep. 2010

[68] P. Davenport et al., "Applying ensemble neural networks to an inverse problem solution to prosthetic socket pressure measurement," in Int. Conf. MEDO, 2016, pp. 1-5.

[69] R. Amali et al., "An artificial intelligence approach for measurement and monitoring of pressure at the residual limb/socket interface - a clinical study," Insight - Non-Destructive Test. Cond. Monit., vol. 50, no. 7, pp. 374-383, Jul. 2008.

[71] M. C. Faustini et al., "An Experimental and Theoretical Framework for Manufacturing Prosthetic Sockets for Transtibial Amputees," IEEE Trans. Neural Syst. Rehabil. Eng., vol. 14, no. 3, pp. 304-310, Sep. 2006.

[72] D. M. Sengeh and H. Herr, "A Variable-Impedance Prosthetic Socket for a Transtibial Amputee Designed from Magnetic Resonance Imaging Data," JPO, vol. 25, no. 3, pp. 129-137, Jul. 2013.

[73] G. N. Nehme, "Pressure Relief in Prosthetic Sockets through Hole Implementation Using Different Materials," World Acad. Sci. Eng. Technol. Int. J. Chem. Mol. Nucl. Mater. Metall. Eng., vol. 9, no. 9, pp. 1118-1122, 2015.

[74] G. Pirouzi et al., "Development of an Air Pneumatic Suspension System for Transtibial Prostheses," Sensors, vol. 14, no. 9, pp. 16754-16765, Sep. 2014

[75] A. El-Sayed et al., "Piezoelectric Bimorphs' Characteristics as InSocket Sensors for Transfemoral Amputees," Sensors, vol. 14, no. 12, pp. 23724-23741, Dec. 2014.

[76] G. Sordo and L. Lorenzelli, "Design of a novel tri-axial force sensor for optimized design of prosthetic socket for lower limb amputees," in Symp. DTIP MEMS/MOEMS, 2016, pp. 1-4.

[77] G. Janusson et al., "Socket liner incorporating sensors to monitor amputee progress," U.S. Patent 7377 944, May 27, 2008.

[78] R. D. Alley, "Method for use of a compression stabilized prosthetic socket interface," U.S. Patent 8323 353, Dec. 4, 2012.

[79] J. Giannatsis and V. Dedoussis, "Additive fabrication technologies applied to medicine and health care: a review," Int. J. Adv. Manuf. Technol., vol. 40, no. 1-2, pp. 116-127, Jan. 2009.

[80] M. Vaezi et al., "A review on 3D micro-additive manufacturing technologies," Int. J. Adv. Manuf. Technol., vol. 67, no. 5-8, pp. 1721-1754, Jul. 2013.

[81] M. Manti et al., "Stiffening in Soft Robotics: A Review of the State of the Art," IEEE Robot. Autom. Mag., vol. 23, no. 3, pp. 93-106, Sep. 2016.

[82] J. E. Sanders et al., "How does adding and removing liquid from socket bladders affect residual-limb fluid volume?," JRRD, vol. 50, no. 6, pp. 845-60, 2013.

[83] W. J. Board et al., "A comparison of trans-tibial amputee suction and vacuum socket conditions," Prosthet. Orthot. Int, vol. 25, no. 3, 
pp. 202-209, Jan. 2001

M. Lilja et al., "Relaxed versus activated stump muscles during casting for trans-tibial prostheses.," Prosthet. Orthot. Int, vol. 23, no. 1, pp. 13-20, Apr. 1999.

[85] N. A. Abd Razak et al., "Comparison study of the prosthetics interface pressure profile of air splint socket and ICRC polypropylene socket for upper limb prosthetics," Biocybern. Biomed. Eng., vol. 35, no. 2, pp. 100-105, 2015.

[86] M. Mercier et al., "Fluidic Flexible Matrix Composites for Volume Management in Prosthetic," ASME Conf., 2014, pp. 1-7.

[87] J. Goswami et al., "Walking in a vacuum assisted socket shifts the stump fluid balance," Prosthet. Orthot. Int, vol. 27, no. 2, pp. 107113, Aug. 2003

[88] J. Uellendahl and S. Mandacina, "Custom silicone sockets for myoelectric prostheses," in MyoElectric Controls/Powered Prosthetics Symposium, Fredericton, 2005, pp. 17-19.

[89] J. E. Sanders and D. V Cassisi, "Mechanical performance of inflatable inserts used in limb prosthetics.," JRRD, vol. 38, no. 4, pp. 365-74.

[90] R. M. Greeenwald et al., "Volume management: smart variable geometry socket (SVGS) technology for lower-limb prostheses," Prosthet. Orthot., vol. 15, no. 3, pp. 107-112, 2003.

[91] M. De Volder and D. Reynaerts, "Pneumatic and hydraulic microactuators: a review," J. Micromech. Microeng., vol. 20, no. 4, pp. 43001, Apr. 2010.

[92] W. Carrigan et al., "Pneumatic actuator inserts for interface pressure mapping and fit improvement in lower extremity prosthetics," in IEEE Int. Conf. BioRob, 2016, pp. 574-579.

[93] V. Phillips, "Socket insert having a bladder system," U. S. Patent 7 655 049, 2010.

[94] M. Love, "Prosthetic socket containing inflatable means," U.S. Patent 5405 405, 1995.

[95] G. Karason, "Artificial limb socket containing volume control pad," U.S. Patent 6923 834, 2005.

[96] B. Ezenwa, "Prosthetic socket with real-time dynamic control of pressure points," U.S. Patent 7670 386, 2010.

[97] B. Wang, C. Zhang, C. Zeng, and L. Kramer, "Prosthetic socket apparatus and systems," U.S. Patent 9486 333, 2016.

[98] S. Phillips et al., "Use of a Dynamic Load Strap in adjustable anatomical suspension for transradial amputations," in Myoelectric Symp., 2011.

[99] A. Nakamura and N. Abe, "Banner advertisement selecting method.," U.S. Patent 6591 248, 2003.

[100] J. A. Bennett Wilson et al., "A Variable Volume Socket for Belowknee Prostheses," Clin. Prosthetics Orthot., vol. 11, no. 1, pp. 1119, 1987.

[101] A. Johnson et al., "Designing for Affordability, Application, and Performance," JPO, vol. 24, no. 2, pp. 80-85, Apr. 2012.

[102] G. Hurley and J. Williams, "Modular prosthetic sockets and methods for making same," U.S. Patent 14/856 267, 2015.

[103] A. Bache and M. Tuttle, "Adjustable prosthetic limb system.," U.S. Patent 9050 202, 2015.

[104] C. King, "Vacuum assisted heat/perspiration removal system and limb volume management for prosthetic device.," U.S. Patent Application 11/518 064, 2006.

[105] J. T. Kahle et al., "Comparative Effectiveness Of An Adjustable Transfemoral Prosthetic Interface Accomodating Volume Fluctuation: Case Study.," Technol. Innov., vol. 18, no. 2-3, pp. 175-183, Sep. 2016.

[106] K. Ghoseiri and M. R. Safari, "Prevalence of heat and perspiration discomfort inside prostheses: Literature review," JRRD, vol. 51, no. 6, pp. 855-868, 2014.

[107] A. Richardson and M. P. Dillon, "User experience of transtibial prosthetic liners: A systematic review," Prosthet. Orthot. Int, vol. 41, no. 1, pp. 6-18, Feb. 2017

[108] K. Hachisuka et al., "Hygiene problems of residual limb and silicone liners in transtibial amputees wearing the total surface bearing socket," Arch. Phys. Med. Rehabil., vol. 82, no. 9, pp. 1286-1290, Sep. 2001.

[109] T. Bertels et al., "Breathable Liner for Transradial Prostheses," in MyoElectric Controls/Powered Prosthetics Symposium Fredericton, 2011.
[110] G. K. Klute et al., "The thermal conductivity of prosthetic sockets and liners," Prosthet. Orthot. Int, vol. 31, no. 3, pp. 292-299, Sep. 2007.

[111] C. Lake and T. Supan, "The Incidence of Dermatological Problems in the Silicone Suspension Sleeve User.," JPO, vol. 9, no. 3, pp. 97106, 1997.

[112] E. Nadel et al., "Importance of skin temperature in the regulation of sweating," J Appl Physiol, vol. 31, no.1, pp. 80-87, 1971.

[113] Y. Han et al., "An automatic and portable prosthetic cooling device with high cooling capacity based on phase change," Appl. Therm. Eng., vol. 104, pp. 243-248, 2016.

[114] K. Ghoseiri et al., "The prototype of a thermoregulatory system for measurement and control of temperature inside prosthetic socket," Prosthet. Orthot. Int, vol. 40, no. 6, pp. 751-755, Dec. 2016.

[115] J. T. Peery et al., "Residual-limb skin temperature in transtibial sockets.," JRRD, vol. 42, no. 2, pp. 147-54.

[116] E. A. Huff et al., "Measuring Residual Limb Skin Temperatures at the Skin-Prosthesis Interface," JPO, vol. 20, no. 4, pp. 170-173, Oct. 2008.

[117] R. Caldwell and S. Fatone, "Technique for Perforating a Prosthetic Liner to Expel Sweat," JPO, vol. 29, no. 3, pp. 145-147, Jul. 2017.

[118] J. Martin and A. Pollock, "Nanotechnological Effects On Prosthetics," Sep. 2016, Available: http://www.apotsfuto.com/2016/09/nanotechnological-effectsonprosthetics.html

[119] M. M. Wernke et al., "SmartTemp Prosthetic Liner Significantly Reduces Residual Limb Temperature and Perspiration," JPO, vol. 27, no. 4, pp. 134-139, Oct. 2015.

[120] C. Lake, "The Evolution of Upper Limb Prosthetic Socket Design," JPO, vol. 20, no. 3, pp. 85-92, Jul. 2008.

[121] Y. Hanet al., "An automatic and portable prosthetic cooling device with high cooling capacity based on phase change," Appl. Therm. Eng., vol. 104, pp. 243-248, Jul. 2016.

[122] K. Ghoseiri et al., "Temperature Measurement and Control System for Transtibial Prostheses: Functional Evaluation," Assist. Technol., pp. 1-8, Oct. 2016.

[123] Y. Hanet al., "A thermal management device for a lower-limb prosthesis," Appl. Therm. Eng., vol. 82, pp. 246-252, May 2015.

[124] C. M. Webber and B. L. Davis, "Design of a novel prosthetic socket: Assessment of the thermal performance," J. Biomech., vol. 48, no. 7, pp. 1294-1299, May 2015.

[125] P. Einarsson, "Ventilated prosthesis system," U.S. Patent 7867 286, 2002.

[126] Pacanowsky, “Adjustable prosthesis,” U.S. Patent 8480 759, 2013.

[127] G. L. Walters, "Prosthesis Cooling System," U.S. Patent Application 14/377 899, 2014.

[128] S. B. Riffat and X. Ma, "Improving the coefficient of performance of thermoelectric cooling systems: a review," Int. J. Energy Res., vol. 28, no. 9, pp. 753-768, Jul. 2004.

[129] J. Zhe and Y. Han, "Low-power method and device for cooling prosthetic limb socket based on phase change," U.S. Patent Application 14/889 162, 2014.

[130] C. King, "Airflow regulation system for artificial limb and associated methods," U.S. Patent 8475 537, 2013.

[131] M. Siman-Tov and J. A. Crabtree, "Personal cooling apparatus and method," U.S. Patent 6276 155, 2001.

[132] D. C. Abeysingheet al., "Novel MEMS pressure and temperature sensors fabricated on optical fibers," J. Micromech. Microeng., vol. 12, no. 3, pp. 229-235, May 2002.

[133] T. Jacksonet al., "Measuring soil temperature and moisture using wireless MEMS sensors," Measurement, vol. 41, no. 4, pp. 381390, May 2008

[134] G. Ciutiet al., "MEMS Sensor Technologies for Human Centred Applications in Healthcare, Physical Activities, Safety and Environmental Sensing: A Review on Research Activities in Italy," Sensors, vol. 15, no. 3, pp. 6441-6468, Mar. 2015.

[135] X. Zhao and R. Liu, "Recent progress and perspectives on the toxicity of carbon nanotubes at organism, organ, cell, and biomacromolecule levels," Environ. Int., vol. 40, pp. 244-255, Apr. 2012 . 